\title{
Harmonic Modeling of a Diode-Clamped Multilevel Voltage Source Converter for Predicting Uncharacteristic Harmonics
}

\author{
Tian Hao Huang and Kuo Lung Lian $(D)$ \\ Department of Electrical Engineering, National Taiwan University of Science and Technology, Taiwan \\ Correspondence should be addressed to Kuo Lung Lian; ryankuolian@gmail.com
}

Received 15 October 2018; Accepted 5 November 2018; Published 5 December 2018

Academic Editor: Vincenzo Vespri

Copyright (c) 2018 Tian Hao Huang and Kuo Lung Lian. This is an open access article distributed under the Creative Commons Attribution License, which permits unrestricted use, distribution, and reproduction in any medium, provided the original work is properly cited.

\begin{abstract}
In response to the growing demand for medium- and high-power trends, multilevel voltage source converters (VSCs) have been attracting growing considerations. One of the widely used VSCs are the diode-clamped multilevel VSC (DCM-VSC). As these converters proliferate, their harmonic impact may become significant. Nevertheless, a harmonic model for the DCM-VSC is currently lacking in the literature. In this paper, the ABCD matrix, mapping the input harmonics to the output harmonics of DCM-VSC, is derived. As the matrix is formulated in the time-domain, the output harmonics are exact and do not suffer from harmonic truncation errors. As the paper will demonstrate, the derived ABCD matrix can be easily applied to a microgrid system and users can easily predict all the uncharacteristic harmonics when a microgrid is subjected to various conditions of imbalance. In addition to all the results being validated with those of PSCAD/EMTDC, the computation time of the proposed method is in contrast much shorter.
\end{abstract}

\section{Introduction}

Voltage source converters (VSCs) have been widely used in the modern power system [1]. They are the main interconnection devices for distributed generators (DGs) and energy storage systems in a microgrid system [2]. At the transmission and distribution levels, they are also the main building blocks of the flexible ac transmission system devices and custom power controllers, respectively. While many benefits may be realized from proliferation of VSCs in the power grid, these come at the expense of generating harmonic distortion. It is therefore essential to obtain accurate harmonic models for VSCs to predict their generated current and voltage harmonic under various conditions and to understand how these harmonics will interact with the rest of the power system.

Electromagnetic transient programs such as PSCAD/ EMTDC can provide accurate harmonic analysis results. However, its disadvantage is that an initialization transient must die out before steady-state is reached when Fourier analysis can be performed [3]. The simulation time step must be sufficiently small for the VSCs to capture the switching dynamics, and this leads to excessively long simulation times. Alternatively, one can rely on harmonic power flow to obtain the harmonic solutions. However, a frequency map (FM) between converters' input and output harmonics usually requires deriving. Hence, the main objective of this paper is to derive the FM of a multilevel VSC.

Depending on the number of the voltage levels, VSCs can be classified into two-level and multilevel (more than twolevel) converters. The multilevel VSCs have the advantages of reduced voltage stress on power semiconductor switches, lower distortions of the input current, and lower $\mathrm{dv} / \mathrm{dt}$, as compared with their two-level counterparts. Consequently, multilevel VSCs have become attractive even for the lowand medium-power range such as in the distributed energy conversion application [4]. Among many various types of multilevel VSCs, diode-clamped multilevel VSCs (DCMVSCs) are the most widely used for utility and high-power industry applications [5] ever since their first appearance in 1981 [6]. As these converters become more dispersed, their harmonic impact on the system could be significant. There have been numerous publications [7-9] addressing harmonic modeling of a two-level VSC. Nevertheless, a generalized 


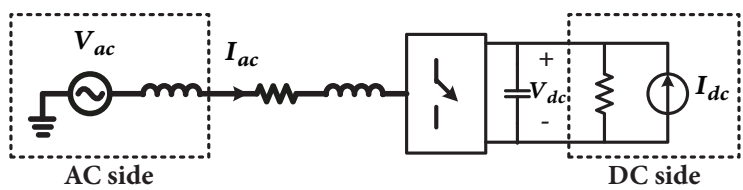

FIGURE 1: Equivalent external network of DCM-VSC.

harmonic model of the DCM-VSC has not been developed yet. Thus, the focus of this paper is on the DCM-VSCs.

As VSCs may be subjected to various power quality disturbance, the derived harmonic models of DCM-VSCs should be general and able to account for various conditions such as unbalanced grid voltage $[10,11]$ and unbalanced dc voltages $[12,13]$. The imbalance in the $\mathrm{dc}$ voltage comes from (i) the DGs with different voltage levels connected to the $\mathrm{dc}$ link of the converters $[14,15]$ and (ii) the inherent $\mathrm{dc}$ voltage imbalance of DCM-VSCs [16]. It will be demonstrated in the paper that the two cases will lead to drastically different harmonic profiles. The first dc voltage imbalance may result in low-order even current harmonics at the ac side [14], which in turn may cause undesired dc component and unequal positive and negative peak values [17]. However, in the second case, no even harmonics are generated, but some uncharacteristic odd harmonics are generated. Moreover, as will be demonstrated in the paper, the harmonic profile of converters will also alter significantly in a microgrid environment or in the presence of neighboring converters. Therefore, it is essential to have an accurate harmonic model, capable of predicting uncharacteristic harmonics under various conditions.

The current models for DCM-VSCs are inadequate for harmonic modeling. Khajehoddin et al. [4] proposed a current flow model for the DCM-VSCs. They formulated the capacitor currents as well as the input dc current as a function of the switching functions and output currents. Nevertheless, they ignored the high-order harmonics of the switching functions, which may result in inaccurate side band harmonic arising with converter modulation. Generalized averaged models of DCM-VSCs have been proposed in [18]. However, all the high-order harmonics are effectively eliminated in averaging operations, thereby producing inaccurate harmonic results. Jayaram et al. [19] derived a five-level DCM-VSC in abc frame for which the switching functions are obtained using curve fitting. However, such a model becomes very complex when the number of level is high. He et al. [20] have modeled the DCM-VSC as a Norton equivalent circuit. However, they have assumed that the capacitors are large enough so that the dc link voltage can be assumed to be constant. Such models are only accurate in predicting characteristic harmonics and are not able to account for the uncharacteristic harmonics under unbalanced conditions.

Based on the work of Larsen et al. [21], the FM of an AC$\mathrm{DC}$ converter is more conveniently modeled by means of an ABCD matrix. A DCM-VSC can be visualized in Figure 1. The harmonic injections caused by external networks can be modeled as ac voltage harmonics $V_{a c}$ (in this paper, capital letters represent harmonic domain variables, whereas lower-case letters represent time-domain variables) and dc current harmonics $I_{d c}$ at the point of common coupling. The converter in turn produces ac current harmonics and dc voltage harmonics $V_{d c}$ via the ABCD matrix as stated in (1).

$$
\left[\begin{array}{c}
I_{a c} \\
V_{d c}
\end{array}\right]=[A B C D]\left[\begin{array}{l}
V_{a c} \\
I_{d c}
\end{array}\right] \text {. }
$$

Therefore, the main goal of this paper is to derive an accurate ABCD matrix for a DCM-VSC under various conditions. The proposed method essentially consists of two steps: the first is to derive the state-space equations of the DCMVSC, and the second is to treat the harmonics of interest as state variables and solve the entire augmented state-space equations piecewise with the periodicity constraint imposed. Then the ABCD matrix is obtained by mapping the resulting submatrix of the state transition matrix to the harmonic domain by a transformation matrix. The advantage of the proposed method is that

(i) calculation times are much shorter than the bruteforce time-domain method as it directly bypasses the transient state;

(ii) different from the harmonic domain methods, there is no need to know how many harmonics need to be included to obtain the harmonics of interest;

(iii) the predicted harmonics are exact and do not suffer from harmonic truncation errors [7];

(iv) it can be easily expanded to study a microgrid system containing multiple numbers of DCM-VSCs.

To validate the proposed model, all the predicted harmonics are verified with those obtained from PSCAD/EMTDC.

The rest of the paper is organized as follows: Section 2 describes the state-space model of a DCM-VSC. Section 3 shows how the harmonic $\mathrm{ABCD}$ coupling matrix is derived. In Section 4, various scenarios of imbalance for a DCMVSC and DCM-based microgrid are studied, and the predicted results are verified with those of PSCAD/EMTDC, demonstrating the validity of the proposed method. Finally, a conclusion is given in Section 5 .

\section{Generalized State-Space Representation of a DCM-VSC}

Different from other conventional multilevel converters such as flying capacitors or cascaded multilevel converters, DCMVSCs have forbidden states [22], in which the output voltage is different if the current direction is different under the same switching function state. Figure 2 shows the three-level VSC where the upper switches are assumed to be complementary with the lower switches in each leg.

To illustrate the forbidden states, Figure 3 is referred to, and without loss of generality, only phase A leg of the VSC is shown. When $s_{11}$ turns on and $s_{12}$ turns off and if the current flows as shown in Figure 3(a), $v_{a o}=-v_{c 2}$. However, when the current flows in the reverse direction, the circuit becomes Figure 3(b) and $v_{a o}=v_{c 1}$. Consequently $s_{11}=1$ and 
TABLE 1: Legitimate switching states of phase A.

\begin{tabular}{lcc}
\hline$s_{11}$ & $s_{12}$ & $v_{a o}$ \\
\hline 0 & 0 & $v_{C 1}$ \\
\hline 0 & 1 & 0 \\
\hline 1 & 1 & $-v_{C 2}$ \\
\hline
\end{tabular}

TABLE 2: Switching status of $s_{a}$.

\begin{tabular}{lccc}
\hline & $s_{a 1}$ & $s_{a 2}$ & $s_{a 3}$ \\
\hline$s_{a} \longrightarrow e_{a 1}$ & 1 & 0 & 0 \\
\hline$s_{a} \longrightarrow e_{a 2}$ & 0 & 1 & 0 \\
\hline$s_{a} \longrightarrow e_{a 3}$ & 0 & 0 & 1 \\
\hline
\end{tabular}

$s_{12}=0$ constitute a forbidden state because the output voltage is different when the current direction is different. In contrast, when $s_{11}=0, s_{12}=1$, the circuit remains that shown in Figure 4, regardless of the direction of current flow. Such as a state is a legitimate state. Table 1 lists all the possible legitimate switching states for phase A.

To have a better representation of all these states, Figure 2 is replaced with Figure 5 where all the bidirectional switches are replaced with single-pole triple-throw switches [23]. $s_{a}$ in Figure 5 can be represented by three switching states, $s_{a 1}$, $s_{a 2}$, and $s_{a 3}$. When $s_{a}$ is connected to $e_{a 1}$ in Figure $5, s_{a 1}=1$, $s_{a 2}=0$, and $s_{a 3}=0$. Table 2 summarizes the switching status for $s_{a}$. Similar rules apply to $s_{b}$ and $s_{c}$.

Based on Table 2, one can derive a generalized m-level ( $m$ is assumed to be odd.), three-phase, four-wire model (see Figure 6) of DCM-VSC with parameters such as inductance and resistance for each phase that may not necessarily be the same. In addition, without loss of generality, the stimuli are assumed to take the form of $u_{n} e^{j\left(n \omega t+\theta_{n}\right)}$, where $n$ is the number of harmonics and $\theta_{n}$ the phase angle at the $n$-th harmonic.

$$
\frac{d}{d t}\left[\begin{array}{l}
x \\
u
\end{array}\right]=\left[\begin{array}{ll}
F & G \\
0 & \Omega
\end{array}\right]\left[\begin{array}{l}
x \\
u
\end{array}\right]
$$

where

$$
\begin{aligned}
x & =\left[\begin{array}{lllllll}
i_{a} & i_{b} & i_{c} & v_{C 1} & c_{C 2} & \cdots & v_{C(m-1)}
\end{array}\right]^{T} \\
u & =\left[\begin{array}{lllll}
v_{s a} & v_{s b} & v_{s c} & i_{L}
\end{array}\right]^{T}
\end{aligned}
$$

Submatrices, $F, G$, and $\Omega$ are stated in the Appendix. Moreover, the derivation of a simple three-level DCM-VSC can also be found in [24].

\section{Derivation of the ABCD Matrix for Harmonic Evaluation}

Equation (2) is a time-domain state-space representation. To step into the harmonic domain, one can treat the harmonics of interest as harmonic states and augment them to (2) by recasting a Fourier integral into a differential equation [7] as stated in (4).

$$
\frac{d x_{h}}{d t}=j n \omega x_{h}+\frac{1}{T} e^{-j n \omega T} x(t)
$$

where $x_{h}$ is the harmonic state, $T$ is the period, and $x_{h}(0)=$ 0 .

If one wants to know the harmonics for the three-phase current and all the $m-1$ capacitor voltages, the overall equation is as stated in (5).

$$
\frac{d}{d t}\left[\begin{array}{l}
x \\
u \\
x_{h}
\end{array}\right]=\breve{F}\left[\begin{array}{l}
x \\
u \\
x_{h}
\end{array}\right]
$$

where

$$
\begin{aligned}
& \breve{F}=\left[\begin{array}{ccc}
F & G & 0 \\
0 & \Omega & 0 \\
D & 0 & J
\end{array}\right] \\
& D=\left[\begin{array}{c}
D_{1} \\
D_{2} \\
\vdots \\
D_{2+m}
\end{array}\right]
\end{aligned}
$$$$
D_{1}=\left[\begin{array}{cccc}
\frac{e^{j n \omega T}}{T} & 0 & \cdots & 0 \\
\frac{e^{j(n-1) \omega T}}{T} & 0 & \cdots & 0 \\
\vdots & \vdots & \vdots & \vdots \\
\frac{1}{T} & 0 & \cdots & 0 \\
\vdots & \vdots & \vdots & \vdots \\
\frac{e^{-j(n-1) \omega T}}{T} & 0 & \cdots & 0 \\
\frac{e^{-j n \omega T}}{T} & 0 & \cdots & 0
\end{array}\right]_{(2 * n+1) \times(2+m)}
$$

$$
D_{2+m}=\left[\begin{array}{cccc}
0 & \cdots & 0 & \frac{e^{j n \omega T}}{T} \\
0 & \cdots & 0 & \frac{e^{j(n-1) \omega T}}{T} \\
\vdots & \vdots & \vdots & \vdots \\
0 & \cdots & 0 & \frac{1}{T} \\
\vdots & \vdots & \vdots & \vdots \\
0 & \cdots & 0 & \frac{e^{-j(n-1) \omega T}}{T} \\
0 & \cdots & 0 & \frac{e^{-j n \omega T}}{T}
\end{array}\right]_{(2 * n+1) \times(2+m)}
$$

$J=\operatorname{diag}\left(J_{1}, J_{2}, \cdots, J_{2+m}\right)$

$J_{1}=J_{2}=\cdots=J_{2+m}=\operatorname{diag}(-j n \omega$,

$$
-j(n-1) \omega, \cdots, 0, \cdots, j(n-1) \omega, j n \omega)
$$


4

Mathematical Problems in Engineering

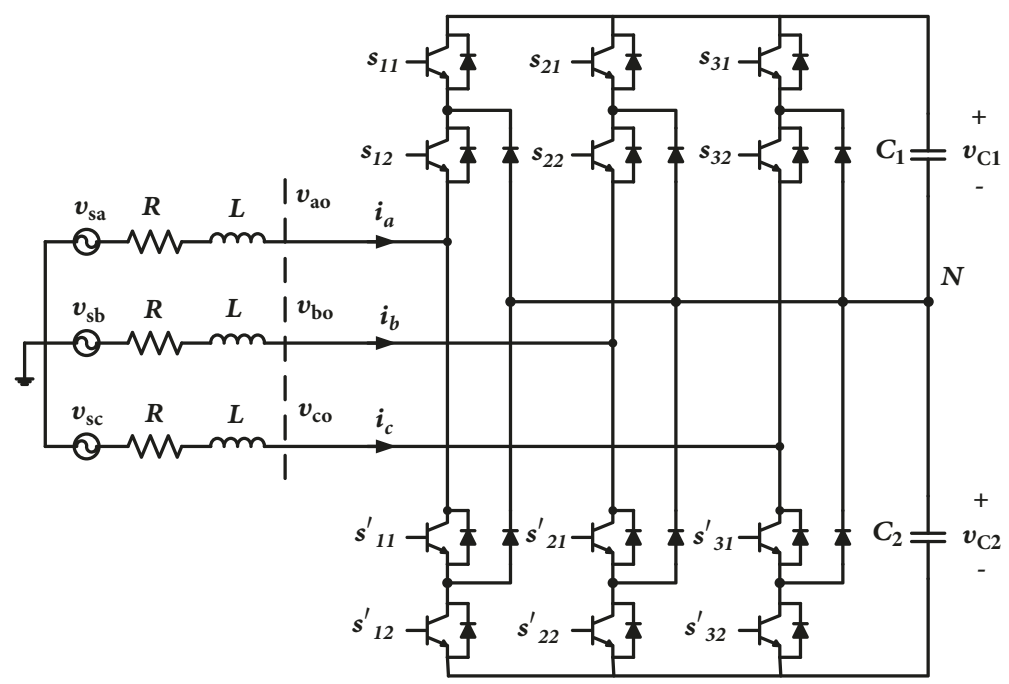

FIGURE 2: Three-phase, three-level DCM-VSC.

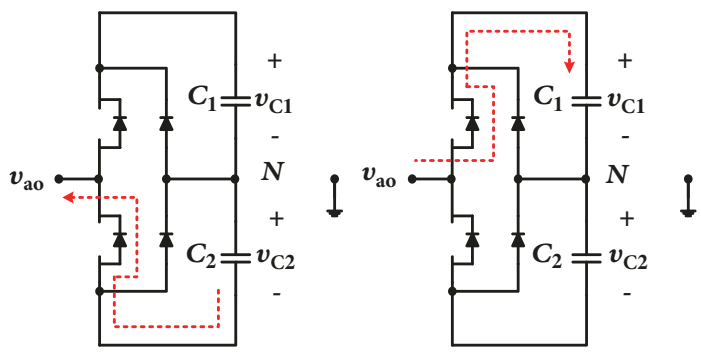

FIGURE 3: DCM-VSC forbidden states: (a) Left: current out of converter; (b) Right: current into the converter.

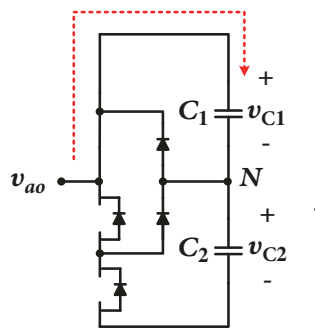

(a)

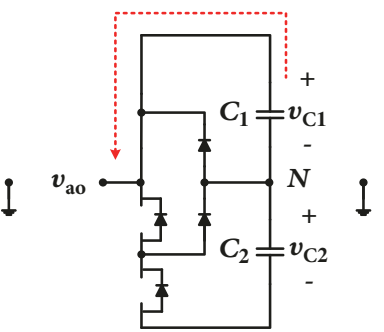

(b)

FIGURE 4: DCM-VSC legitimate states: (a) current out of converter; (b) current into the converter.

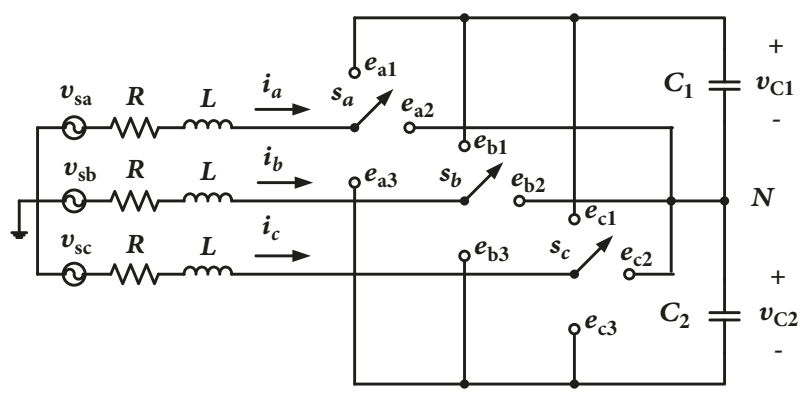

Figure 5: The equivalent single-pole, triple-throw model for a three-level DCM-VSC. 


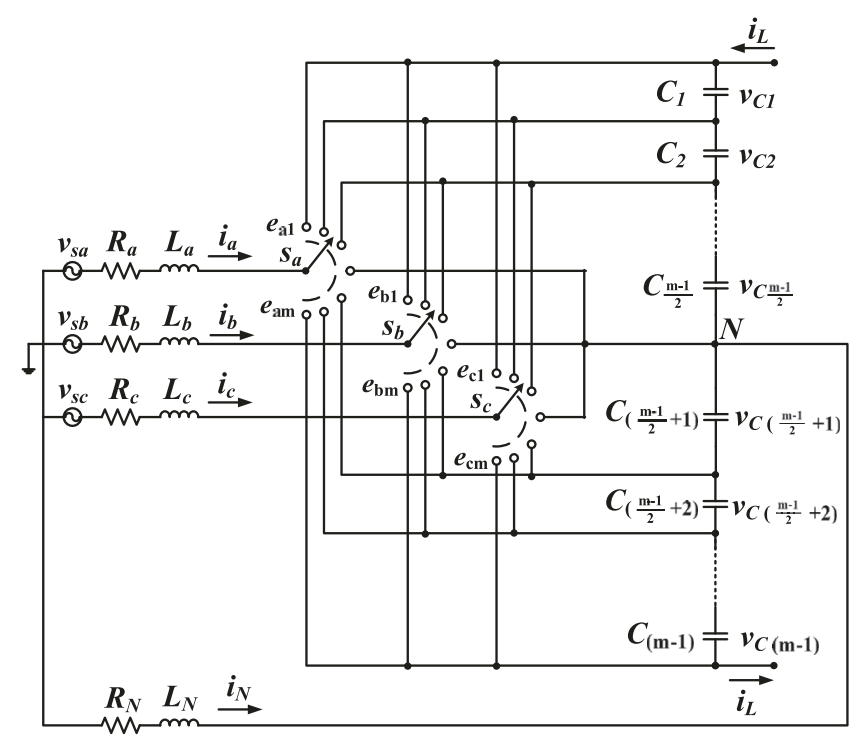

FIGURE 6: The equivalent single-pole, m-throw model for a three-phase, four-wire m-level DCM-VSC.

Evaluating (5) over $T$, one gets (7).

$$
\left[\begin{array}{c}
x(T) \\
u(T) \\
x_{h}(T)
\end{array}\right]=\check{\Phi}\left[\begin{array}{c}
x(0) \\
u(0) \\
x_{h}(0)
\end{array}\right]
$$

where

$$
\check{\Phi}=e^{\check{F}_{n_{s w}}\left(T-t_{n_{s w}}\right)} \cdots e^{\check{F}_{2}\left(t_{2}-t_{1}\right)} e^{\check{F}_{1}\left(t_{1}-0\right)},
$$

$n_{s w}$ is the number of switching times.

As proved in [7], $\check{\Phi}$ in general takes the following form.

$$
\check{\Phi}=\left[\begin{array}{lll}
\alpha & \beta & 0 \\
0 & \gamma & 0 \\
\varsigma & \eta & \xi
\end{array}\right]
$$

Imposing the periodicity constraints

$$
x(t+T)=x(t)
$$

where $t$ is generally set to 0 and the values of $x_{h}(T)$ are essentially the harmonics of interests [7]. In other words, the output harmonics, such as AC current harmonics $I_{a b c}$ and DC voltage harmonics $V_{d c}$, are equal to $x_{h}(T)$ and can be obtained analytically as stated in (11).

$$
\left[\begin{array}{c}
I_{a b c} \\
V_{d c}
\end{array}\right]=[M]\left[\begin{array}{c}
v_{s a b c}(0) \\
i_{d c}(0)
\end{array}\right]
$$

where

$$
[M]=\left\{\varsigma\left[(I-\alpha)^{-1} \beta\right]+\eta\right\}
$$

The matrix $M$ in (11) relates the steady-state initial conditions of the ac voltages and dc currents (i.e., $v_{s a b c}(0)$ and $\left.i_{d c}(0)\right)$ to the ac current harmonics $I_{a c}$ and dc voltage harmonics $V_{d c}$. Because the input is the time-domain variable, one needs to find the relation between input steady-state initial conditions $\left(v_{s a b c}(0)\right.$ and $\left.\left.i_{d c}(0)\right)\right)$ and input harmonics (i.e., $V_{s a b c}$ and $\left.I_{d c}\right)$. As the stimuli are in the form of $u_{n} e^{j\left(n \omega t+\theta_{n}\right)}$,

$$
\left[\begin{array}{c}
V_{s a b c} \\
I_{a b c}
\end{array}\right]=\left[\begin{array}{c}
v_{s a b c}(0) \\
i_{d c}(0)
\end{array}\right] \text {. }
$$

Hence,

$$
\left[\begin{array}{c}
I_{a b c} \\
V_{d c}
\end{array}\right]=[A B C D]_{a b c}\left[\begin{array}{c}
V_{s a b c} \\
I_{d c}
\end{array}\right],
$$

where $[A B C D]_{a b c}=[M]$. Alternatively, one can also formulate (14) in terms of the sequential components (zero, positive, and negative sequence components are designated as 0,1 , and 2 , respectively). Since

$$
\begin{gathered}
I_{a b c}=P \cdot I_{012}, \\
V_{s a b c}=P \cdot V_{s 012}
\end{gathered}
$$

where

$$
\begin{aligned}
& P=\left[\begin{array}{ccc}
1 & 1 & 1 \\
1 & a^{2} & a \\
1 & a & a^{2}
\end{array}\right] \\
& a=1 \angle 120^{\circ} .
\end{aligned}
$$

Substituting (15) into (16) and converting harmonic phase currents $I_{a b c}$ into harmonic sequential currents $I_{012}$, one obtains

$$
\left[\begin{array}{ll}
P & 0 \\
0 & I
\end{array}\right]\left[\begin{array}{c}
I_{012} \\
V_{d c}
\end{array}\right]=[A B C D]_{a b c}\left[\begin{array}{cc}
P & 0 \\
0 & I
\end{array}\right]\left[\begin{array}{c}
V_{a 012} \\
I_{d c}
\end{array}\right]
$$




$$
\left[\begin{array}{l}
I_{012} \\
V_{d c}
\end{array}\right]=[A B C D]_{012}\left[\begin{array}{c}
V_{012} \\
I_{d c}
\end{array}\right],
$$

where $[A B C D]_{012}=\left[\begin{array}{ll}P & 0 \\ 0 & I\end{array}\right]^{-1}[A B C D]_{a b c}\left[\begin{array}{ll}P & 0 \\ 0 & I\end{array}\right]$ and $I$ is the identity matrix.

\section{Case Studies}

To validate the uncharacteristic harmonics predicted by the proposed method, five case studies are investigated. In the first case, the characteristic harmonics produced by a threelevel DCM-VSC under a balanced ac grid are first evaluated. Then, the uncharacteristic harmonics due to the negative sequence ac voltage injection are studied. The second case is to investigate the uncharacteristic harmonics of a three-level DCM-VSC with unequal dc voltage levels, caused by external DGs. The third case is to show that the proposed model can reproduce the inherent unbalanced dc voltage of a five-level DCM-VSC. In the fourth case, the proposed model is shown to be able to predict the uncharacteristic harmonics arising from an imbalance in system parameters. The converter parameters are extracted from [25] and listed in Table 3. Note that for Cases 1 to $3, R_{N}$ and $L_{N}$ are assumed to be infinity. Thus, a three-phase, three-wire DCM-VSC system is investigated. In contrast, $R_{N}$ and $L_{N}$ for Case 4 are finite, and a three-phase, four-wire DCM-VSC system is investigated. Finally, in case 5, a microgrid consisting of the converters presented in Cases 2, 3, and 4 is modeled using the proposed method.

The modulation strategy for multilevel converters can be classified into three types, specifically, carrier-based pulse width modulation (PWM), space vector modulation, and selective harmonic elimination. Among them, the carrierbased modulation strategy is widely used in industry [26]. The carrier-based PWM can be further classified into phaseshifted PWM (PS-PWM) and level-shifted PWM (LS-PWM). PS-PWM is rarely used for DCM-VSC as it has no modular structure and the carrier cannot be associated with a particular cell or treated independently [27]. The LS-PWM, on the other hand, can be easily adapted to a DCM. There are three types of LS-PWMs [28]-(i) in-phase disposition (IPD), where all carrier are in phase; (ii) alternative phase opposite disposition (APOD), where all carriers are alternatively in opposite disposition; and (iii) phase opposite disposition (POD), where all the positive carriers are in phase with each other and in opposite phase of the negative carriers. For the case studies, the IPD-LS-PWM is used because of its better power quality [28]. The switching times are obtained by solving the transcendental equations of the modulating and carrier signals.

4.1. Case 1: Balanced and Unbalanced AC Grid (Negative Sequence Voltage Injection). The three-level DCM-VSC is first connected to a balanced AC grid. Figure 7 shows the maximum difference $\left(I_{d}\right)$ of the ac currents obtained by the proposed method and those by PSCAD/EMTDC under various time steps. As the figure shows, the simulation step $t_{\text {step }}$ needs to reduce to $0.1 \mu \mathrm{s}$ in order for PSCAD/EMTDC model to reach the same level of accuracy as the proposed

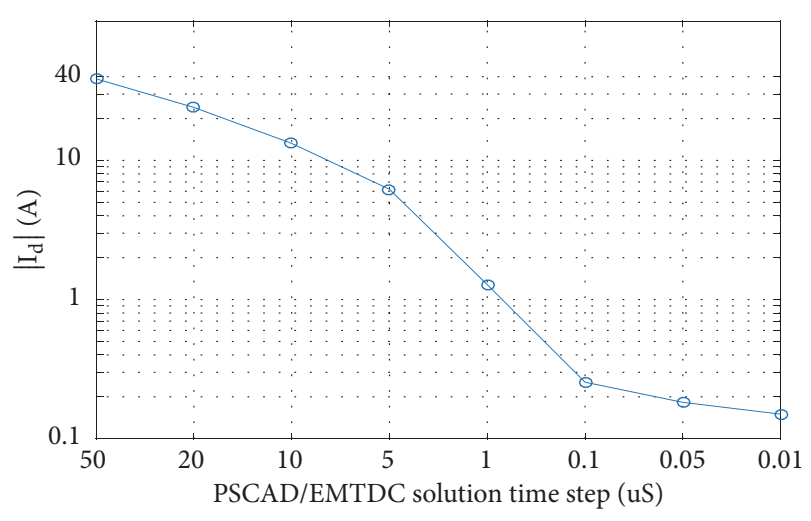

FIgURE 7: $\left|I_{d}\right|$ versus solution time step.

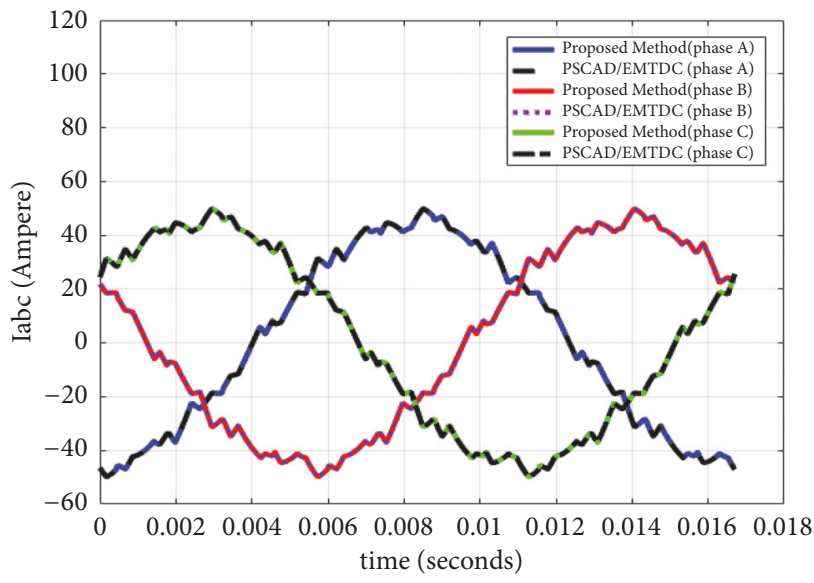

FIGURE 8: Balanced steady-state AC current waveforms.

method. Figure 8 shows the resulting balanced three-phase currents for both PSCAD/EMTDC $\left(t_{\text {step }}=0.1 \mu \mathrm{s}\right)$ and the proposed method. Nevertheless, the calculation time for PSCAD/EMTDC with time step of $0.1 \mu \mathrm{s}$ is about 85 times longer than the proposed method. Figure 9 shows the current harmonic spectra in terms of its positive and negative sequence components. It clearly shows that under balanced condition, the DCM-VSC only produces characteristic harmonics.

It is to be noted that input stimuli in (2) can be easily extended to other stimuli such as the cosine function by means of Euler's formula, which is stated in (20).

$$
\cos (\omega t+\theta)=\frac{e^{j(\omega t+\theta)}+e^{-j(\omega t+\theta)}}{2}
$$

The following, as an example, are the step-by-step procedures for calculating the positive sequence of the fundamental current and dc voltage harmonic under the balanced condition:

(1) The input stimuli for phases $\mathrm{A}, \mathrm{B}$, and $\mathrm{C}$ are $\left\{\left(v_{s}\right)\right.$ $\left.2) e^{-j(\omega t+\theta)},\left(v_{s} / 2\right) e^{j(\omega t+\theta)}\right\}, \quad\left\{\left(v_{s} / 2\right) e^{-j(\omega t+\theta-2 \pi / 3)},\left(v_{s} /\right.\right.$ $\left.2) e^{j(\omega t+\theta-2 \pi / 3)}\right\}$, and $\left\{\left(v_{s} / 2\right) e^{-j(\omega t+\theta+2 \pi / 3)},\left(v_{s} /\right.\right.$ 2) $\left.e^{j(\omega t+\theta+2 \pi / 3)}\right\}$. Note that $v_{s}$ is the magnitude of the source voltage. 
TABLE 3: The parameter list of the three-level DCM-VSC.

\begin{tabular}{lccc}
\hline Parameter & Value & Parameter & Value \\
\hline$R_{a}, R_{b}, R_{c}$ & $0.1 \Omega$ & firing angle, $\delta$ & $0^{\circ}$ \\
\hline$L_{a}, L_{b}, L_{c}$ & $0.004 \mathrm{H}$ & modulation index, $m_{a}$ & \\
\hline$f$ & $60 \mathrm{~Hz}$ & modulation factor, $m_{f}$ & 0.833 \\
\hline$C_{1}, C_{2}$ & $1000 \mu \mathrm{F}$ & source voltage & 15 \\
\hline
\end{tabular}

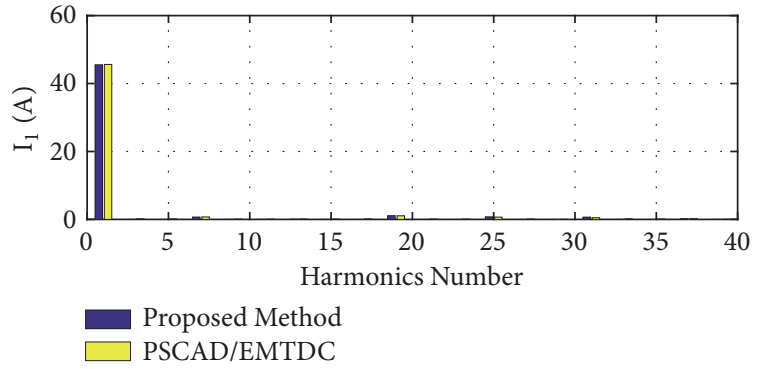

(a)

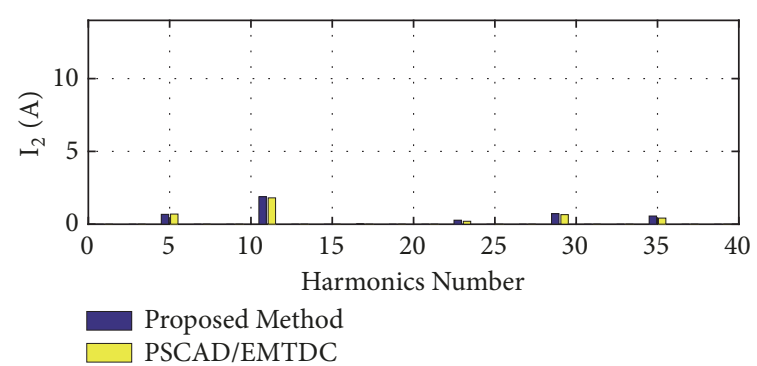

(b)

FIgURE 9: (a) The harmonic spectra of ac currents of Figure 8 in terms of positive sequence components; (b) the harmonic spectra of ac currents of Figure 8 in terms of negative sequence components.

(2) Substitute the parameter values listed in Table 3 into the form, similar to (5)

(3) Evaluate (7) to obtain $\check{\Phi}$.
(4) Extract the submatrices, $A, B, C$, and $D$, which are as follows.

$$
\begin{aligned}
& \text { A } \\
& =\left[\begin{array}{cccccc}
1.6490+0.0489 j & 1.6709-0.2624 j & -0.7822-1.4525 j & -1.0626+1.5416 j & -0.8668+1.4036 j & -0.6083-1.2792 j \\
-0.7822-1.4525 j & -0.6083-1.2792 j & -0.8668+1.4036 j & 1.6709+0.2624 j & 1.6490+0.0489 j & -1.626+1.5416 j \\
-0.8668+1.4036 j & -1.0626+1.5416 j & 1.6490+0.0489 j & -0.6083-1.2792 j & -0.7822-1.4525 j & 1.6709-0.2624 j
\end{array}\right] \\
& B=\left[\begin{array}{c}
0.1887+0.0148 j \\
-0.1072+0.1560 j \\
-0.0815-0.1708 j
\end{array}\right] \\
& C=\left[\begin{array}{llllll}
5.8125-0.3137 j & 5.8125+0.3137 j & -3.178-4.8769 j & -3.178+4.8769 j & -2.6345+5.1907 j & -2.6345-5.1907 j \\
5.8125-0.3137 j & 5.8125+0.3137 j & -3.178-4.8769 j & -3.178+4.8769 j & -2.6345+5.1907 j & -2.6345-5.1907 j
\end{array}\right] \\
& D=\left[\begin{array}{l}
0 \\
0
\end{array}\right]
\end{aligned}
$$

(5) The ac current and dc voltage harmonics can be obtained by evaluating (14). Note that the ac current harmonics need to be multiplied by two to obtain the correct phasor forms

$$
\left[\begin{array}{c}
I_{a}^{1} \\
I_{b}^{1} \\
I_{c}^{1} \\
V_{c 1}^{0} \\
V_{c 2}^{0}
\end{array}\right]=\left[\begin{array}{lllll}
2 & 0 & 0 & 0 & 0 \\
0 & 2 & 0 & 0 & 0 \\
0 & 0 & 2 & 0 & 0 \\
0 & 0 & 0 & 1 & 0 \\
0 & 0 & 0 & 0 & 1
\end{array}\right]\left[\begin{array}{c}
22.76-0.34 \mathrm{j} \\
11.09+19.88 \mathrm{j} \\
11.67-19.54 \mathrm{j} \\
292.03 \\
292.03
\end{array}\right]
$$

$$
=\left[\begin{array}{c}
-45.53-0.68 \mathrm{j} \\
22.18+39.77 \mathrm{j} \\
23.35-39.09 \mathrm{j} \\
292.03 \\
292.03
\end{array}\right]
$$

where $I_{a}^{1}, I_{b}^{1}$, and $I_{c}^{1}$ denote the fundamental currents of phases $\mathrm{A}, \mathrm{B}$, and $\mathrm{C}$, respectively, and $V_{c 1}^{0}$ and $V_{c 2}^{0}$ are the two dc voltages across $C_{1}$ and $C_{2}$ 


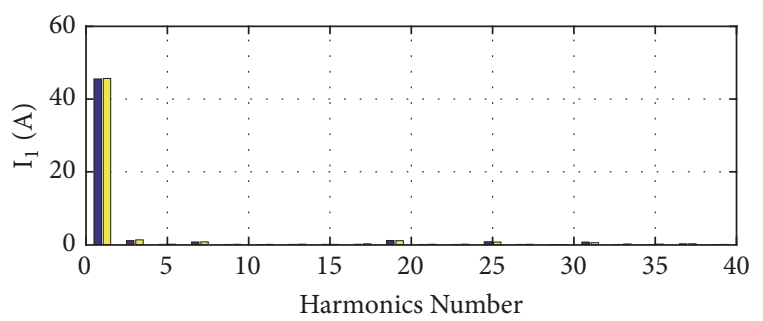

Proposed Method PSCAD/EMTDC

(a)

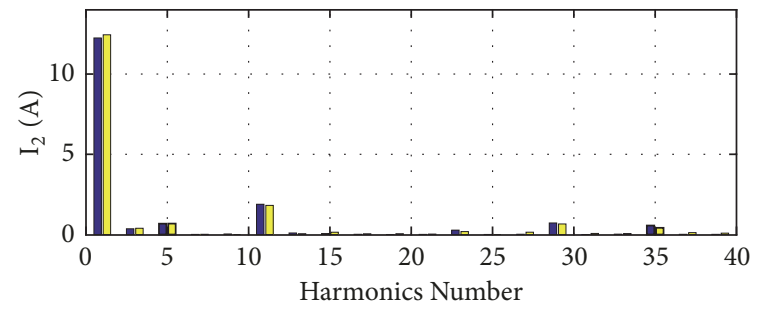

Proposed Method PSCAD/EMTDC

(b)

FIGURE 10: Unbalanced ac grid case: (a) the harmonic spectra of ac currents of in terms of positive sequence components; (b) the harmonic spectra of ac currents of in terms of negative sequence components.

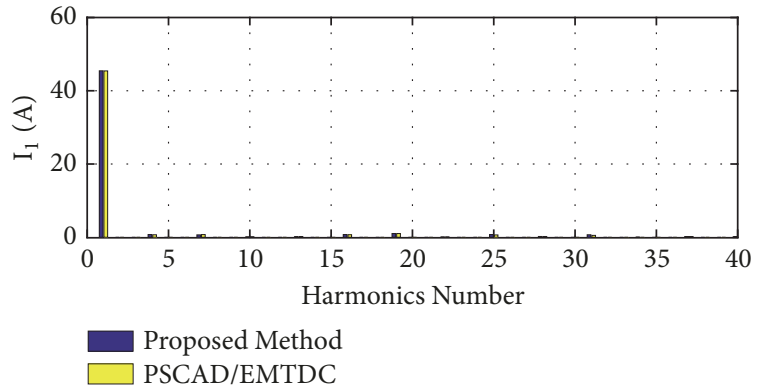

(a)

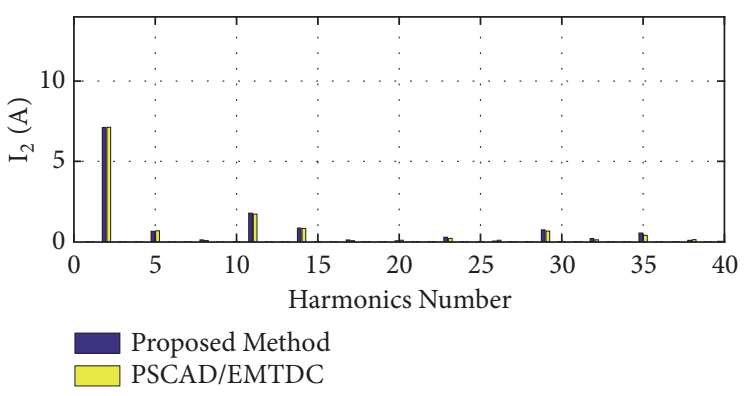

(b)

Figure 11: Case 2: (a) the harmonic spectra of ac currents in terms of positive sequence components; (b) the harmonic spectra of ac currents in terms of negative sequence components.

(6) Evaluate (19), and the fundamental positive $\left(I_{1}^{1}\right)$, negative $\left(I_{2}^{1}\right)$, and zero sequence $\left(I_{0}^{1}\right)$ currents and the two dc voltages are stated as (26).

$$
\left[\begin{array}{c}
I_{0}^{1} \\
I_{1}^{1} \\
I_{2}^{1} \\
V_{c 1}^{0} \\
V_{c 2}^{0}
\end{array}\right]=\left[\begin{array}{c}
0 \\
45.53-0.68 \mathrm{j} \\
0 \\
292.03 \\
292.03
\end{array}\right]
$$

Next, a $5 \%$ negative sequence of the fundamental voltage is added to the AC grid, and the voltage takes the following form.

$$
\begin{aligned}
& V_{s a}=V_{s} e^{j \omega t}+0.05 V_{s} e^{j \omega t} \\
& V_{s b}=V_{s} e^{j \omega t-120^{\circ}}+0.05 V_{s} e^{j \omega t+120^{\circ}} \\
& V_{s c}=V_{s} e^{j \omega t+120^{\circ}}+0.05 V_{s} e^{j \omega t-120^{\circ}}
\end{aligned}
$$

Figure 10 shows the harmonic spectra of this unbalanced case in terms of positive and negative sequence components. In this instance, the uncharacteristic harmonics such as the negative sequence fundamental and third harmonics are accurately predicted by the proposed method.
4.2. Case 2: Unequal DC Voltage due to DGs with Different Voltage Levels. In this case, we try to simulate the case where the split dc link capacitors of the DCM-VSC can be controlled independently. A commonly seen situation is to have a different PV panel connected to each capacitor, with each panel being controlled independently to achieve its own point of maximum power [14]. Such a case leads to unequal dc voltage level for different capacitors with respect to the neutral point, $\mathrm{N}$. To simulate such a case, two DC sources with $v_{D C 1}=350 \mathrm{~V}$ connected in parallel with $C_{1}$ and $v_{D C 1}=$ $230 \mathrm{~V}$ connected in parallel with $C_{2}$ are connected to the dc link. The resulting current spectra, shown in Figure 11, feature undesired negative sequence even-order current harmonics such as the second and 14-th harmonics. The values of these harmonics are accurately predicted by the proposed method when compared with those of PSCAD/EMTDC.

4.3. Case 3: Inherent Unbalanced Capacitor Voltage for A 5-Level DCM-VSC. Different from the previous case, this case study investigates a five-level DCM-VSC. The parameter settings are the same as those in Table 3. As stated in [16, 29], when the level number is greater than 3 , an inherent dc voltage imbalance becomes apparent. In addition, as demonstrated in [22], if the condition

$$
m_{a} \cos (\delta)>0.55
$$




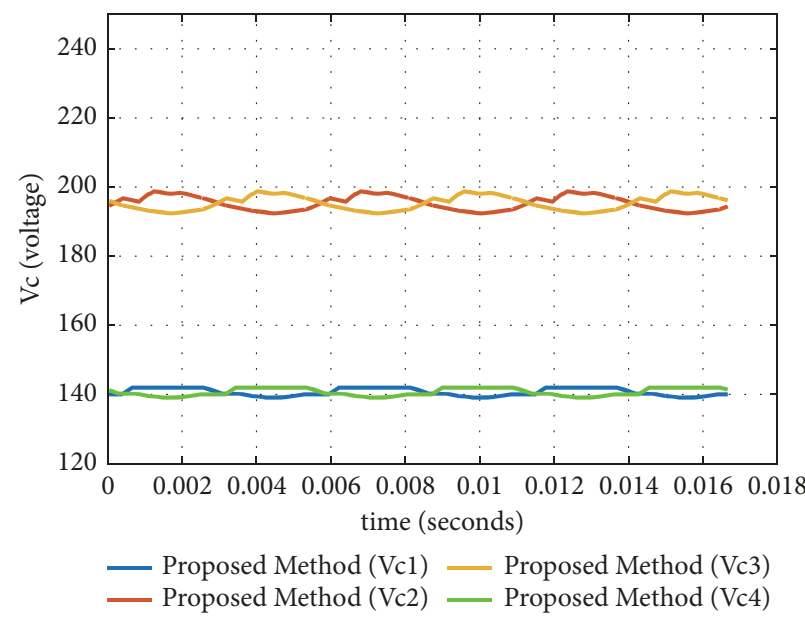

(a)

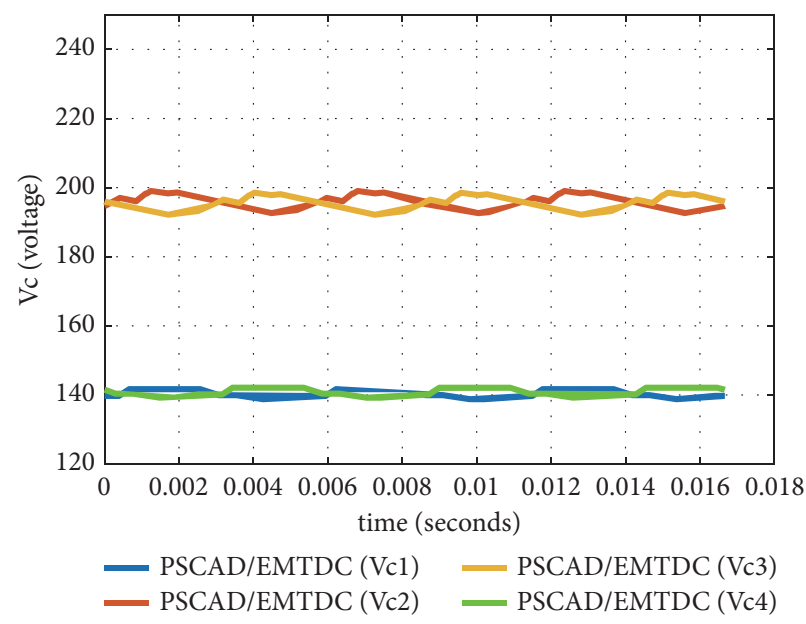

(b)

FIGURE 12: Case 3: (a) the capacitor voltage waveforms obtained by the proposed method; (b) the capacitor voltage waveforms obtained by the PSCAD/EMTDC.

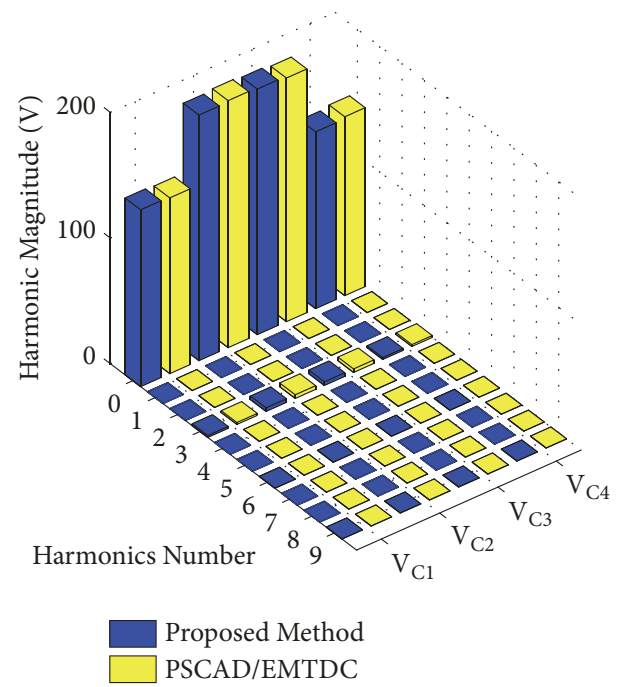

FIGURE 13: Harmonic spectra of each capacitor in the five-level DCM-VSC.

is satisfied, no balancing strategy exists to balance the capacitor voltages. Hence, for the chosen $m_{a}$ and $\delta$ in Table 3, voltage imbalance among capacitors is expected.

Figures 12(a) and 12(b) show the DC voltage waveforms for each capacitor (in this paper, the capacitor labels follow those indicated in Figure 6), obtained by the proposed method and PSCAD/EMTDC. Their corresponding harmonic spectra are in great agreement as shown in Figure 13. Figure 14 shows the current waveforms of the five-level DCM-VSC. Figure 15 shows the corresponding current spectra. Compared with Figure 9, one can see that the positive sequence 7-th, 13-th, 19-th, and 25-th harmonics are much greater for the 5-level DCM-VSC. Moreover, for the negative sequence, the value of the 5 -th harmonic is about 5 times larger than that of 11-th harmonic for the 5-level case, whereas the 11-th harmonic for the 3-level converter is 3 times

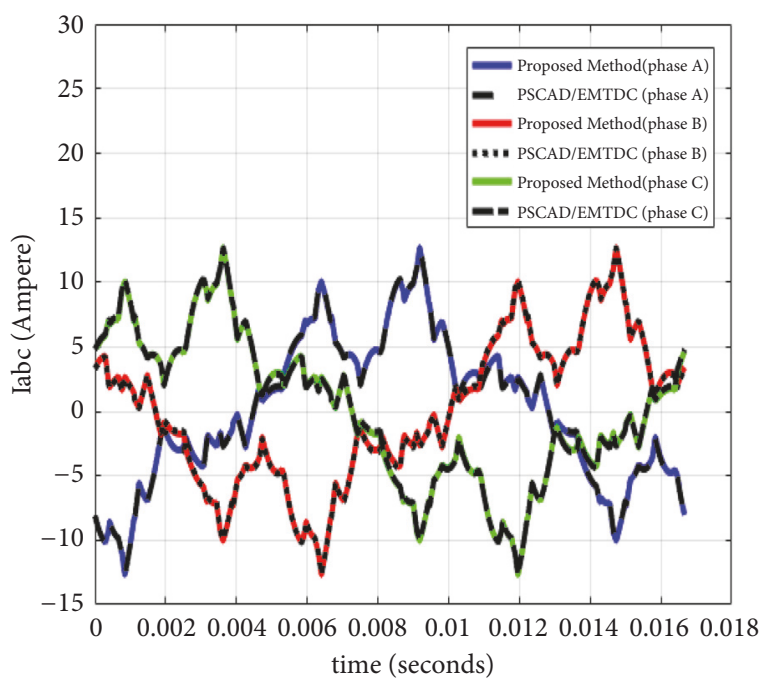

FIGURE 14: Steady-state AC current waveforms of five-level DCMVSC.

higher than that of the 5-th harmonic as seen in Figure 9. Nevertheless, different from Case 2, there is no even current harmonic generated at the ac side. The reason is that, with respect to $\mathrm{N}$, there exists a symmetry between the upper and lower capacitor voltages. In other words, $\left|v_{c 1}\right|=\left|v_{c 4}\right|$ and $\left|v_{c 2}\right|=\left|v_{c 3}\right|$. Consequently, such a symmetry with respect to $\mathrm{N}$ will not generally lead to any even current harmonics at the ac side.

4.4. Case 4: Unbalanced Parameters of a Three-Phase, FourWire DCM-VSC. In this case, we investigate a three-phase, four-wire DCM-VSC system. $R_{N}$ and $L_{N}$ are set equal to $R_{b}$ and $L_{b}$, respectively. The inductance of phase $\mathrm{A}, L_{a}$, is increased by $20 \%$ whereas other parameters remain fixed. Figure 16 shows the portion of $[A B C D]_{012}$, which relates the positive sequence input voltage harmonics $V_{s 1}$ to the zero 


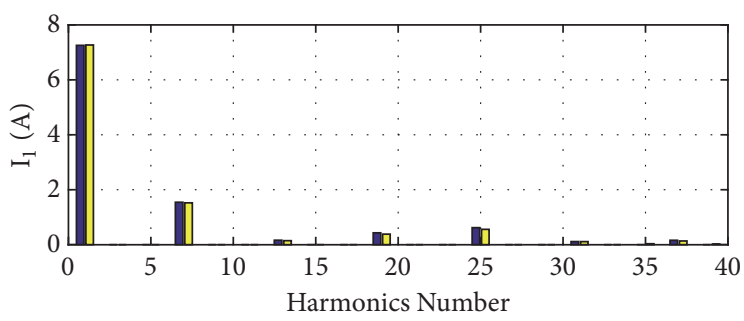

Proposed Method PSCAD/EMTDC

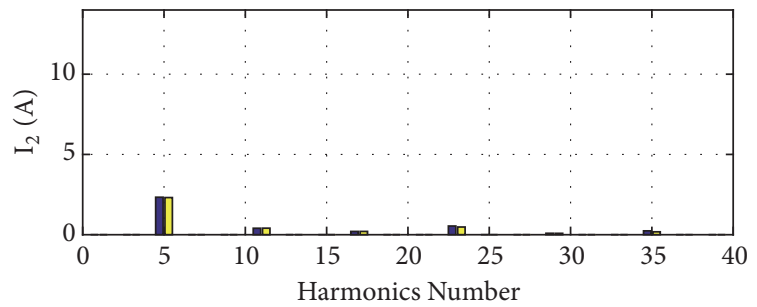

Proposed Method PSCAD/EMTDC

(a)

(b)

Figure 15: Case 3: (a) the current harmonic spectra of five-level DCM-VSC in terms of positive sequence components; (b) the current harmonic spectra of five-level DCM-VSC in terms of negative sequence components.

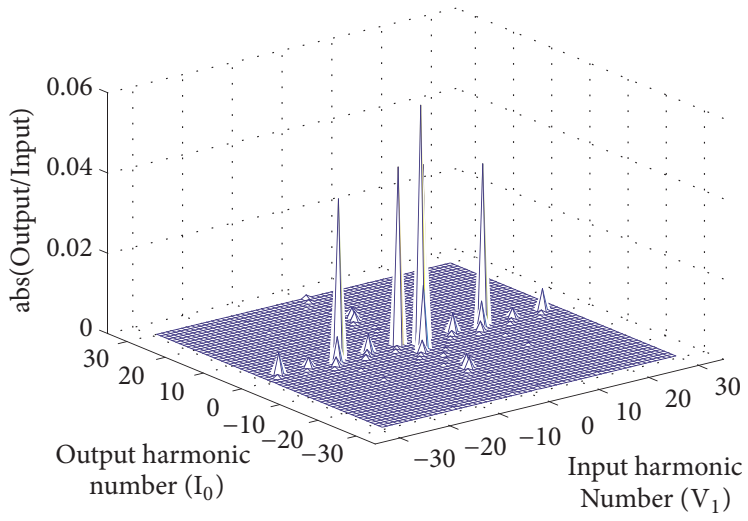

FIGURE 16: The ABCD matrix, relating $V_{s 1}$ to $I_{0}$.

sequence output current harmonics $I_{0}$. Figure 17 shows the ac current harmonic spectra in terms of the positive, negative, and zero sequence components. The figure clearly shows the negative sequence second harmonic due to the unbalanced inductance values. In addition, it also clearly indicates that the derived ABCD matrix can accurately predict the zero sequence current harmonic at various frequency, and their values are in strong agreement with those obtained by PSCAD/EMTDC.

4.5. Case 5: A Multiconverter Unbalanced Microgrid System. Figure 18 shows a microgrid system, consisting of multiple VSCs, which is similar to the Aichi microgrid system in Japan [30]. With the aid of the proposed ABCD matrix, the overall microgrid formulation can be derived.

From Figure 18, one can see the following.

$$
\begin{aligned}
V_{p c c} & =V_{s}-Z_{s} I_{s} \\
I_{s} & =I_{v s c 1}+I_{v s c 2}+I_{v s c 3}+\cdots+I_{v s c n}
\end{aligned}
$$

Also, each converter can be expressed as

$$
\left[\begin{array}{l}
I_{v s c k} \\
V_{d c k}
\end{array}\right]=\left[\begin{array}{ll}
A_{k} & B_{k} \\
C_{k} & D_{k}
\end{array}\right]\left[\begin{array}{c}
V_{p c c} \\
I_{d c k}
\end{array}\right],
$$

where $k=1,2,3, \cdots, n$.
Combining (29), (30), and (31), one can get (32).

$$
\left[\begin{array}{c}
I_{s} \\
V_{d c 1} \\
V_{d c 2} \\
\vdots \\
V_{d c n}
\end{array}\right]=[M]^{-1}[N]\left[\begin{array}{c}
V_{s} \\
I_{d c 1} \\
I_{d c 2} \\
\vdots \\
I_{d c n}
\end{array}\right]
$$

where

$$
\begin{aligned}
& M=\left[\begin{array}{ccccc}
I+\left(A_{1}+A_{2}+\cdots+A_{n}\right) Z_{s} & 0 & 0 & \cdots & 0 \\
C_{1} Z_{s} & I & 0 & \cdots & 0 \\
C_{2} Z_{s} & 0 & I & \cdots & 0 \\
\vdots & \vdots & \vdots & \ddots & 0 \\
C_{n} Z_{s} & 0 & 0 & \cdots & I
\end{array}\right], \\
& N=\left[\begin{array}{ccccc}
A_{1}+A_{2}+\cdots+A_{n} & B_{1} & B_{2} & \cdots & B_{n} \\
C_{1} & D_{1} & 0 & \cdots & 0 \\
C_{2} & 0 & D_{2} & \cdots & 0 \\
\vdots & \vdots & \vdots & \ddots & 0 \\
C_{n} & 0 & 0 & \cdots & D_{n}
\end{array}\right]
\end{aligned}
$$




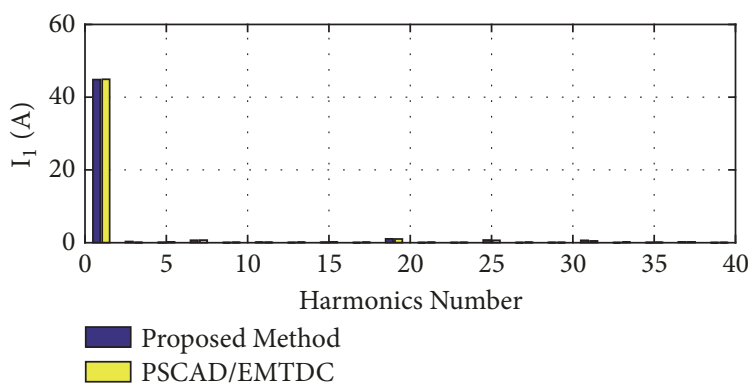

(a)

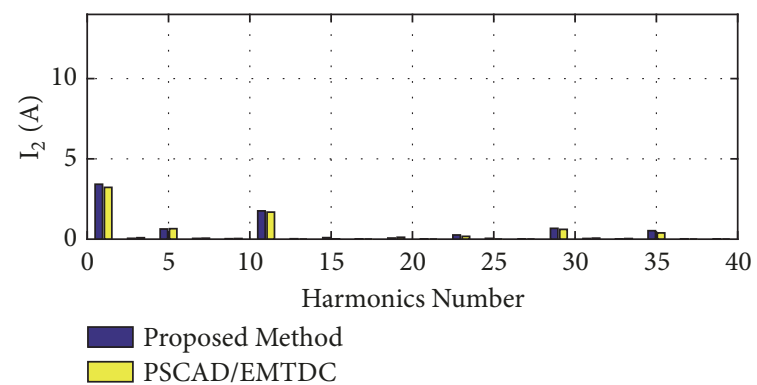

(b)

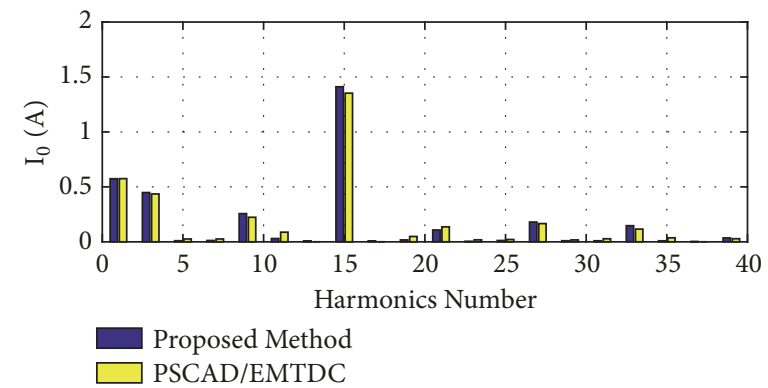

(c)

FIGURE 17: Case 4: (a) the current harmonic spectra of in terms of positive sequence components; (b) the current harmonic spectra of in terms of negative sequence components; (c) the current harmonic spectra of in terms of zero sequence components.

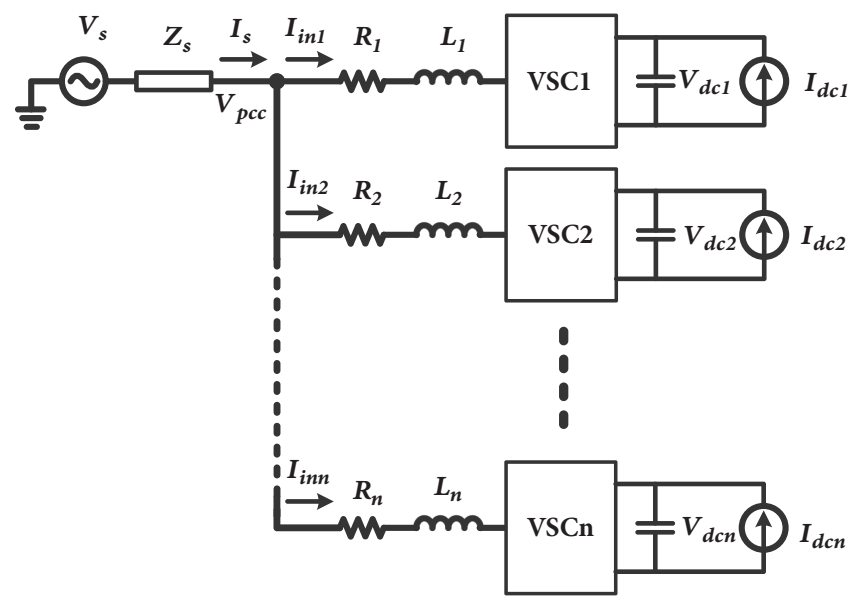

FIGURE 18: A multiconverter microgrid system.

Figure 19 shows a multi-DCM-VSC microgrid system. Assume that the ac grid is unbalanced and takes the form of (27). VSC1 in Figure 19 is a three-level DCM-VSC, having unequal DC voltage due to DGs with different voltage level, as described in Case 2. VSC2 is a 5-level DCM-VSC with inherent unbalanced capacitor voltage as in Case 3. Finally, VSC3 is a three-level VSC with unbalanced parameters as delineated in Case 4. Employing (32), one can obtain the ac currents and capacitor voltages for each converter. Figures 20, 21, and 22 show the ac current spectra for VSC1, VSC2, and VSC3, respectively. To show that the presence of the neighboring converters can have a significant impact on the harmonic spectra of the converter, we resimulate Case 4 with the grid voltage taking the form of (27), and its current spectra are shown in Figure 23. Comparing Figure 22 with Figure 23, one can see that VSC3 exhibits a positive sequence, negative sequence, and zero sequence of the fundamental current of $45.5 \mathrm{~A}, 7.7 \mathrm{~A}$, and $1.7 \mathrm{~A}$, respectively, when it is the only converter connected to the grid. On the other hand, those components reduce to $37 \mathrm{~A}, 5.3 \mathrm{~A}$, and 1.22 A, which correspond to $18 \%, 31 \%$, and $26 \%$ reduction, respectively, under the microgrid environment. Moreover, as can be observed from Figures 22(b) and 23(b), one can notice that a negative sequence of second harmonic is injected from 


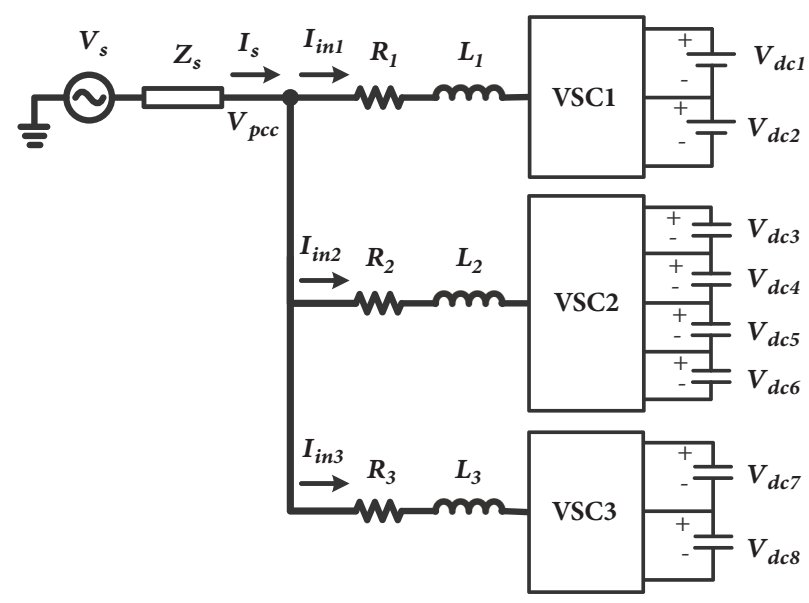

FIGURE 19: An unbalanced microgrid.

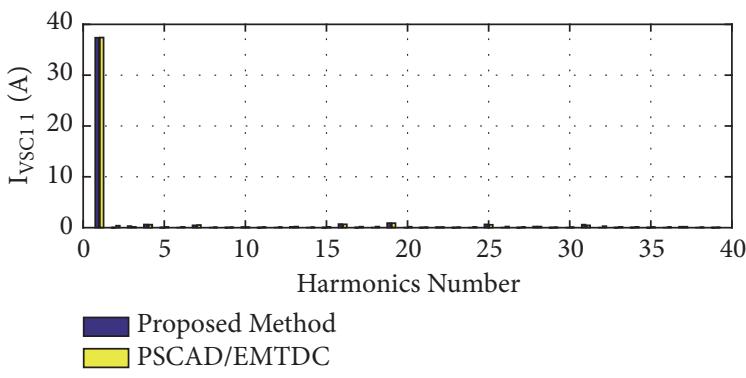

(a)

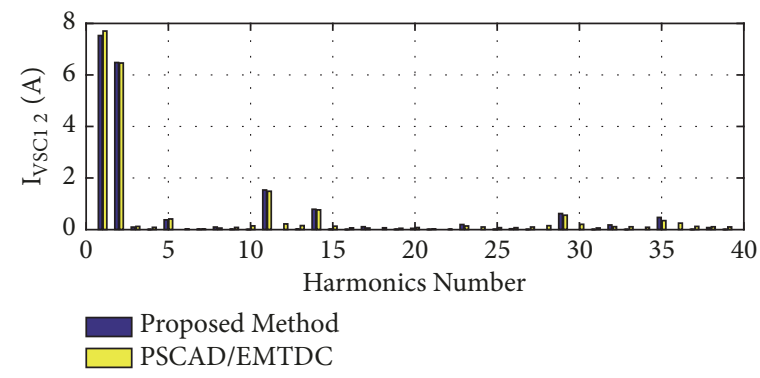

(b)

FIGURE 20: Current spectra of VSC1: (a) positive sequence of $I_{v s c 1}$; (b) negative sequence of $I_{v s c 1}$.

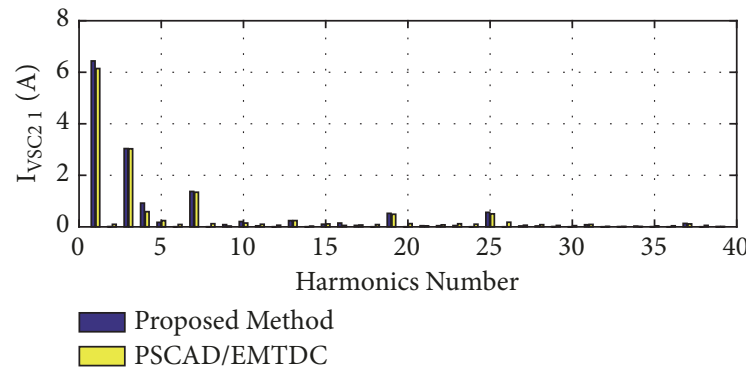

(a)

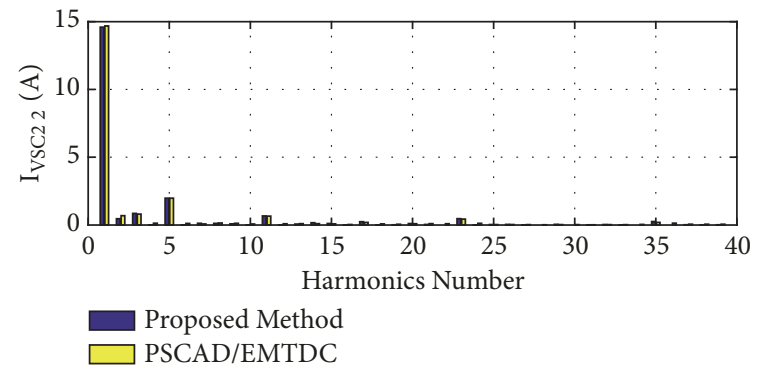

(b)

FIGURE 21: Current spectra of VSC2: (a) positive sequence of $I_{v s c 2}$; (b) negative sequence of $I_{v s c 2}$.

other converters under the microgrid environment whereas no even harmonics exist in Figure 23(b). All the results predicted by the proposed method are consistent with those of PSCAD/EMTDC.

\section{Conclusion}

An AC-DC converter has been more conveniently characterized by the $\mathrm{ABCD}$ matrix for harmonic modeling and analysis. This paper presented a time-domain-based $\mathrm{ABCD}$ matrix for a diode-clamped multilevel voltage source converter. The derived model produced accurate predictions of characteristic and all the uncharacteristic harmonics for various unbalanced conditions. Also, the method yielded accurate results and does not suffer from harmonic truncation errors. Moreover, all the predicted results are in great agreement with those obtained by PSCAD/EMTDC with the calculation time being much shorter. 


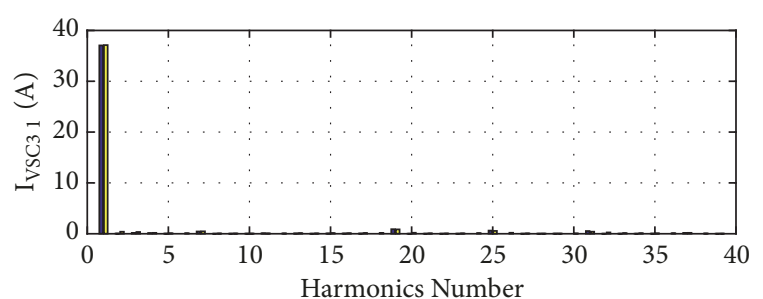

Proposed Method PSCAD/EMTDC

(a)

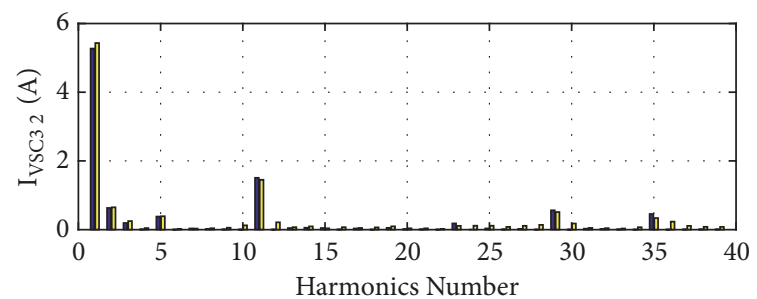

Proposed Method PSCAD/EMTDC

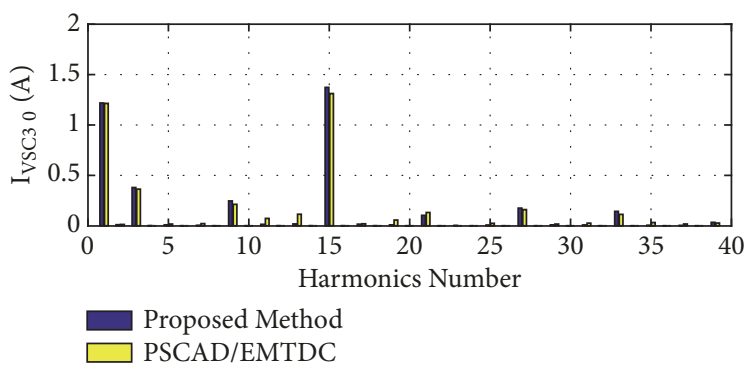

(c)

FIGURE 22: Current spectra of VSC3: (a) positive sequence of $I_{v s c 3}$; (b) negative sequence of $I_{v s c 3}$; (c) zero sequence of $I_{v s c 3}$.

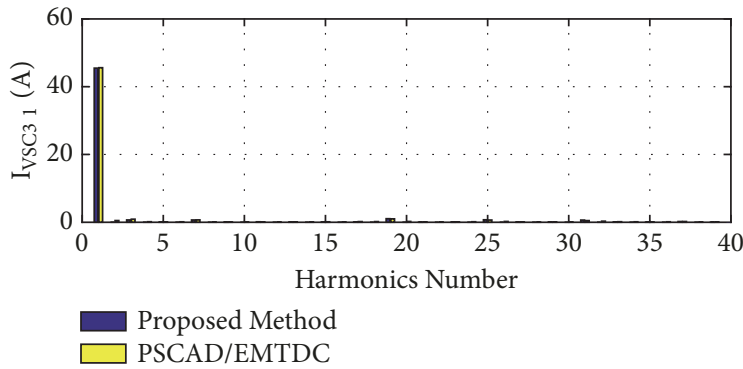

(a)

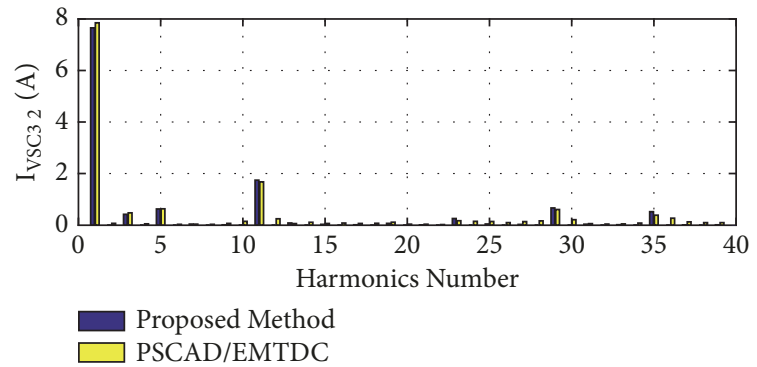

(b)

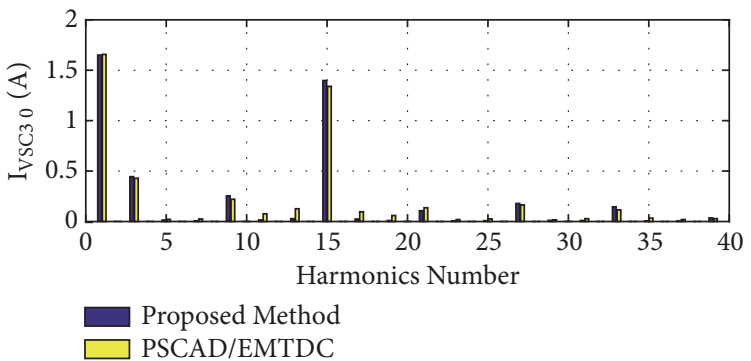

(c)

FIGURE 23: Only VSC3 is connected to the unbalanced ac grid: (a) positive sequence of $I_{v s c 3}$; (b) negative sequence of $I_{v s c 3}$; (c) zero sequence of $I_{v s c 3}$.

\section{Appendix}

Expressions of Submatrices $\Omega, F, G$

$$
\begin{aligned}
& \Omega=\operatorname{diag}\left(J_{1}, J_{2}, J_{3}, J_{4}\right) \\
& F=\left[\begin{array}{cc}
F_{1} & F_{2} \\
F_{3} & 0
\end{array}\right]
\end{aligned}
$$




$$
\begin{aligned}
& F_{1}= {\left[\begin{array}{lll}
-\frac{R_{a} L_{a a}+R_{N} L_{b} L_{c}}{L_{t}} & \frac{R_{b} L_{c} L_{N}-R_{N} L_{b} L_{c}}{L_{t}} & \frac{R_{c} L_{b} L_{N}-R_{N} L_{b} L_{c}}{L_{t}} \\
\frac{R_{a} L_{c} L_{N}-R_{N} L_{a} L_{c}}{L_{t}} & -\frac{R_{b} L_{b b}+R_{N} L_{a} L_{c}}{L_{t}} & \frac{R_{c} L_{a} L_{N}-R_{N} L_{a} L_{c}}{L_{t}} \\
\frac{R_{a} L_{b} L_{N}-R_{N} L_{a} L_{b}}{L_{t}} & \frac{R_{b} L_{a} L_{N}-R_{N} L_{a} L_{b}}{L_{t}} & -\frac{R_{c} L_{c c}+R_{N} L_{a} L_{b}}{L_{t}}
\end{array}\right] } \\
& F_{2}=\left[\begin{array}{lll}
F_{21} & F_{22}
\end{array}\right] \\
& F_{21}=\left[\begin{array}{llll}
\frac{-L_{a a} \Gamma_{a 1}+L_{c} L_{N} \Gamma_{b 1}+L_{b} L_{N} \Gamma_{c 1}}{L_{t}} & \frac{-L_{a a} \Gamma_{a 2}+L_{c} L_{N} \Gamma_{b 2}+L_{b} L_{N} \Gamma_{c 2}}{L_{t}} & \cdots & \frac{-L_{a a} \Gamma_{a \varphi}+L_{c} L_{N} \Gamma_{b \varphi}+L_{b} L_{N} \Gamma_{c \varphi}}{L_{t}} \\
\frac{L_{c} L_{N} \Gamma_{a 1}-L_{b b} \Gamma_{b 1}+L_{a} L_{N} \Gamma_{c 1}}{L_{t}} & \frac{L_{c} L_{N} \Gamma_{a 2}-L_{b b} \Gamma_{b 2}+L_{a} L_{N} \Gamma_{c 2}}{L_{t}} & \cdots & \frac{L_{c} L_{N} \Gamma_{a \varphi}-L_{b b} \Gamma_{b \varphi}+L_{a} L_{N} \Gamma_{c \varphi}}{L_{t}} \\
\frac{L_{b} L_{N} \Gamma_{a 1}+L_{a} L_{N} \Gamma_{b 1}-L_{c c} \Gamma_{c 1}}{L_{t}} & \frac{L_{b} L_{N} \Gamma_{a 2}+L_{a} L_{N} \Gamma_{b 2}-L_{c c} \Gamma_{c 2}}{L_{t}} & \cdots & \frac{L_{a} L_{N} \Gamma_{a \varphi}+L_{a} L_{N} \Gamma_{b \varphi}-L_{c c} \Gamma_{c \varphi}}{L_{t}}
\end{array}\right]
\end{aligned}
$$

$F_{22}$

$$
=\left[\begin{array}{llll}
\frac{L_{a a} \Lambda_{a(\varphi+2)}-L_{c} L_{N} \Lambda_{b(\varphi+2)}-L_{b} L_{N} \Lambda_{c(\varphi+2)}}{L_{t}} & \frac{L_{a a} \Lambda_{a(\varphi+3)}-L_{c} L_{N} \Lambda_{b(\varphi+3)}-L_{b} L_{N} \Lambda_{c(\varphi+3)}}{L_{t}} & \cdots & \frac{L_{a a} \Lambda_{a m}-L_{c} L_{N} \Lambda_{b m}-L_{b} L_{N} \Lambda_{c m}}{L_{t}} \\
\frac{-L_{c} L_{N} \Lambda_{a(\varphi+2)}+L_{b b} \Lambda_{b(\varphi+2)}-L_{a} L_{N} \Lambda_{c(\varphi+2)}}{L_{t}} & \frac{-L_{c} L_{N} \Lambda_{a(\varphi+3)}+L_{b b} \Lambda_{b(\varphi+3)}-L_{a} L_{N} \Lambda_{c(\varphi+3)}}{L_{t}} & \cdots & \frac{-L_{c} L_{N} \Lambda_{a m}+L_{b b} \Lambda_{b m}-L_{a} L_{N} \Lambda_{c m}}{L_{t}} \\
\frac{-L_{b} L_{N} \Lambda_{a(\varphi+2)}-L_{a} L_{N} \Lambda_{b(\varphi+2)}+L_{c c} \Lambda_{c(\varphi+2)}}{L_{t}} & \frac{-L_{b} L_{N} \Lambda_{a(\varphi+3)}-L_{a} L_{N} \Lambda_{b(\varphi+3)}+L_{c c} \Lambda_{c(\varphi+3)}}{L_{t}} & \cdots & \frac{-L_{a} L_{N} \Lambda_{a m}-L_{a} L_{N} \Lambda_{b m}+L_{c c} \Lambda_{c m}}{L_{t}}
\end{array}\right]
$$$$
F_{3}=\left[\begin{array}{ccc}
\frac{\Gamma_{a 1}}{C_{1}} & \frac{\Gamma_{b 1}}{C_{1}} & \frac{\Gamma_{c 1}}{C_{1}} \\
\frac{\Gamma_{a 2}}{C_{2}} & \frac{\Gamma_{b 2}}{C_{2}} & \frac{\Gamma_{c 2}}{C_{2}} \\
\vdots & \vdots & \vdots \\
\frac{\Gamma_{a \varphi}}{C_{\varphi}} & \frac{\Gamma_{b \varphi}}{C_{\varphi}} & \frac{\Gamma_{c \varphi}}{C_{\varphi}} \\
-\frac{\Lambda_{(\varphi+2)}}{C_{(\varphi+1)}} & -\frac{\Lambda_{b(\varphi+2)}}{C_{(\varphi+1)}} & -\frac{\Lambda_{c(\varphi+2)}}{C_{(\varphi+1)}} \\
-\frac{\Lambda_{a(\varphi+3)}}{C_{(\varphi+2)}} & -\frac{\Lambda_{b(\varphi+3)}}{C_{(\varphi+2)}} & -\frac{\Lambda_{c(\varphi+3)}}{C_{(\varphi+2)}} \\
\vdots & \vdots & \vdots \\
-\frac{\Lambda_{a m}}{C_{(m-1)}} & -\frac{\Lambda_{b m}}{C_{(m-1)}} & -\frac{\Lambda_{c m}}{C_{(m-1)}}
\end{array}\right]
$$

$$
\Gamma_{j k}=\sum_{i=1}^{k} S_{j i}
$$$$
\Lambda_{j k}=\sum_{i=k}^{m} S_{j i}
$$$$
j=a, b, c
$$$$
G=\left[\begin{array}{cccc}
G_{a a} & G_{a b} & G_{a c} & 0 \\
G_{b a} & G_{b b} & G_{b c} & 0 \\
G_{c a} & G_{c b} & G_{c c} & 0 \\
0 & 0 & 0 & G_{C 1} \\
0 & 0 & 0 & G_{C 2} \\
\vdots & \vdots & \vdots & \vdots \\
0 & 0 & 0 & G_{C(m-1)}
\end{array}\right]
$$

$G_{a a}=\frac{L_{a a}}{L_{t}} \times\left[\begin{array}{lllllll}1 & 1 & \cdots & 1 & \cdots & 1 & 1\end{array}\right]$

$G_{b b}=\frac{L_{b b}}{L_{t}} \times\left[\begin{array}{lllllll}1 & 1 & \cdots & 1 & \cdots & 1 & 1\end{array}\right]$ 


$$
\begin{aligned}
& G_{c c}=\frac{L_{c c}}{L_{t}} \times\left[\begin{array}{lllllll}
1 & 1 & \cdots & 1 & \cdots & 1 & 1
\end{array}\right] \\
& G_{a b}=-\frac{L_{c} L_{N}}{L_{t}} \times\left[\begin{array}{lllllll}
1 & 1 & \cdots & 1 & \cdots & 1 & 1
\end{array}\right] \\
& G_{a c}=-\frac{L_{b} L_{N}}{L_{t}} \times\left[\begin{array}{lllllll}
1 & 1 & \cdots & 1 & \cdots & 1 & 1
\end{array}\right] \\
& G_{b c}=-\frac{L_{a} L_{N}}{L_{t}} \times\left[\begin{array}{lllllll}
1 & 1 & \cdots & 1 & \cdots & 1 & 1
\end{array}\right] \\
& G_{b a}=G_{a b}, \\
& G_{c a}=G_{a c} \text {, } \\
& G_{c b}=G_{b c} \\
& G_{C 1}=\frac{1}{C_{1}} \times\left[\begin{array}{lllllll}
1 & 1 & \cdots & 1 & \cdots & 1 & 1
\end{array}\right] \\
& G_{C 2}=\frac{1}{C_{2}} \times\left[\begin{array}{lllllll}
1 & 1 & \cdots & 1 & \cdots & 1 & 1
\end{array}\right] \\
& G_{C(m-1)}=\frac{1}{C_{(m-1)}} \times\left[\begin{array}{lllllll}
1 & 1 & \cdots & 1 & \cdots & 1 & 1
\end{array}\right] \\
& \varphi=\frac{m-1}{2} \\
& L_{a a}=L_{b} L_{c}+L_{b} L_{N}+L_{c} L_{N} \\
& L_{b b}=L_{a} L_{c}+L_{a} L_{N}+L_{c} L_{N} \\
& L_{c c}=L_{a} L_{b}+L_{a} L_{N}+L_{b} L_{N} \\
& L_{t}=L_{a} L_{b} L_{c}+L_{a} L_{b} L_{N}+L_{a} L_{c} L_{N}+L_{b} L_{c} L_{N}
\end{aligned}
$$

\section{Data Availability}

The data used to support the findings of this study are available from the corresponding author upon request.

\section{Conflicts of Interest}

The authors declare that there are no conflicts of interest regarding the publication of this paper.

\section{Acknowledgments}

The authors would like to sincerely thank the editor and anonymous reviewers for their valuable comments and suggestions, which improved the quality of the paper. This work was financially supported by the Ministry of Science and Technology (under contract No. 106-2221-E-011-100) and by the Taiwan Building Technology Center from The Featured Areas Research Center Program within the framework of the Higher Education Sprout Project by the Ministry of Education in Taiwan.

\section{References}

[1] J. Segundo-Ramírez, R. Peña-Gallardo, A. Medina, C. NúñezGutiérrez, and N. Visairo-Cruz, "A Comprehensive Modeling of a Three-Phase Voltage Source PWM Converter," Mathematical Problems in Engineering, vol. 2015, Article ID 426245, 11 pages, 2015.

[2] V. Valouch, J. Škramlík, Z. Muller et al., "Power Control at Grid Connected Converters and Analytical Solution of Steady States," Mathematical Problems in Engineering, vol. 2015, Article ID 601916, 12 pages, 2015.

[3] R. K. Subroto and K. L. Lian, "Modeling of a multilevel voltage source converter using the fast time-domain method," IEEE Journal of Emerging and Selected Topics in Power Electronics, vol. 2, no. 4, pp. 1117-1126, 2014.

[4] S. A. Khajehoddin, A. Bakhshai, and P. K. Jain, "A simple voltage balancing scheme for $\mathrm{m}$-level diode-clamped multilevel converters based on a generalized current flow model," IEEE Transactions on Power Electronics, vol. 23, no. 5, pp. 2248-2259, 2008.

[5] A. Yazdani and R. Iravani, "Dynamic model and control of the NPC-based Back-to-Back HVDC system," IEEE Transactions on Power Delivery, vol. 21, no. 1, pp. 414-424, 2006.

[6] A. Nabae, I. Takahashi, and H. Akagi, "A new neutral-pointclamped PWM inverter," IEEE Transactions on Industry Applications, vol. 17, no. 5, pp. 518-523, 1981.

[7] P. W. Lehn and K. L. Lian, "Frequency coupling matrix of a voltage-source converter derived from piecewise linear differential equations," IEEE Transactions on Power Delivery, vol. 22, no. 3, pp. 1603-1612, 2007. 
[8] E. Acha and M. Madrigal, Power System Harmonics: Computer Modelling and Analysis, vol. 1, Wiley, 2001.

[9] C. Collins, N. Watson, and A. Wood, "UPFC modeling in the harmonic domain," IEEE Transactions on Power Delivery, vol. 21, no. 2, pp. 933-938, 2006.

[10] C. F. Nascimento, E. H. Watanabe, O. Diene et al., "Analysis of Noncharacteristic Harmonics Generated by Voltage-Source Converters Operating under Unbalanced Voltage," IEEE Transactions on Power Delivery, vol. 32, no. 2, pp. 951-961, 2017.

[11] Q. Zhong, L. Lin, G. Wang, Y. Zhang, and Z. Wu, "Harmonic analysis model for voltage source converter under unbalanced conditions," IET Generation, Transmission \& Distribution, vol. 9, no. 1, pp. 12-21, 2015.

[12] S. Busquets-Monge, A. Filba-Martinez, S. Alepuz, and A. CallePrado, "A Modulation Strategy to Operate Multilevel Multiphase Diode-Clamped and Active-Clamped DC-AC Converters at Low Frequency Modulation Indices with DC-Link Capacitor Voltage Balance," IEEE Transactions on Power Electronics, vol. 32, no. 10, pp. 7521-7533, 2017.

[13] Y. Wang, H. Liu, W. Wang, and K. Wang, "A Novel NeutralPoint Potential Balance Strategy for Three-Level NPC Back-toBack Converter Based on the Neutral-Point Current Injection Model," Mathematical Problems in Engineering, vol. 2015, Article ID 736828, 12 pages, 2015.

[14] U.-M. Choi, F. Blaabjerg, and K.-B. Lee, "Control Strategy of Two Capacitor Voltages for Separate MPPTs in Photovoltaic Systems Using Neutral-Point-Clamped Inverters," IEEE Transactions on Industry Applications, vol. 51, no. 4, pp. 3295-3303, 2015.

[15] M. M. Hashempour, M.-Y. Yang, and T.-L. Lee, "A DPWMcontrolled three-level T-type inverter for photovoltaic generation considering unbalanced neutral-point voltage," in Proceedings of the 9th Annual IEEE Energy Conversion Congress and Exposition, ECCE '17, pp. 3856-3862, 2017.

[16] M. Saeedifard, R. Iravani, and J. Pou, "Control and DCcapacitor voltage balancing of a space vector-modulated fivelevel STATCOM," IET Power Electronics, vol. 2, no. 3, pp. 203215, 2009.

[17] Y. Liu and G. T. Heydt, "Power system even harmonics and power quality indices," Electric Power Components and Systems, vol. 33, no. 8, pp. 833-844, 2005.

[18] A. Yazdani and R. Iravani, "A generalized state-space averaged model of the three-level NPC converter for systematic DCvoltage-balancer and current-controller design," IEEE Transactions on Power Delivery, vol. 20, no. 2, pp. 1105-1114, 2005.

[19] N. Jayaram, P. Agarwal, and S. Das, "Mathematical model of five-level ac/dc converter in abc reference frame," International Journal of Electrical Power \& Energy Systems, vol. 62, pp. 469475, 2014.

[20] Z. He, H. Hu, Y. Zhang, and S. Gao, "Harmonic resonance assessment to traction power-supply system considering train model in China High-Speed Railway," IEEE Transactions on Power Delivery, vol. 29, no. 4, pp. 1735-1743, 2014.

[21] E. V. Larsen, D. H. Baker, and J. C. McIver, "Low-order harmonic interactions on AC/DC systems," IEEE Transactions on Power Delivery, vol. 4, no. 1, pp. 493-501, 1989.

[22] A. A. Gonzalez, S. A. Verne, and M. I. Valla, Multilevel Converters for Industrial Applications, vol. 7, CRC Press, 2013.

[23] M. Chaves, E. Margato, J. F. Silva, and S. F. Pinto, "Generalized state-space modeling for $\mathrm{m}$ level diode-clamped multilevel converters," Mathematical Methods in Engineering, pp. 67-85, 2014.
[24] T.-H. Huang, Harmonic Modeling of a Diode-Clamped Multilevel Voltage Source Converters for Predicting Noncharacteristic Harmonics [Master thesis], National Taiwan University of Science and Technology, 2018.

[25] Z. Jinghua, M. Yongqing, L. Zhengxi, and L. Kun, "Research on control method of three-level NPC voltage source rectifier," in Proceedings of the 2008 IEEE Vehicle Power and Propulsion Conference, VPPC 2008, pp. 1-8, 2008.

[26] S. Kouro, M. Malinowski, K. Gopakumar et al., "Recent advances and industrial applications of multilevel converters," IEEE Transactions on Industrial Electronics, vol. 57, no. 8, pp. 2553-2580, 2010.

[27] B. M. Wilamowski and J. D. Irwin, Eds., Power Electronics and Motor Drives, CRC Press, 2017.

[28] B. Wu, High-Power Converters and AC Drive, IEEE Press, 2006.

[29] N. Hatti, Y. Kondo, and H. Akagi, "Five-level diode-clamped PWM converters connected back-to-back for motor drives," IEEE Transactions on Industry Applications, vol. 44, no. 4, pp. 1268-1276, 2008.

[30] N. W. A. Lidula and A. D. Rajapakse, "Microgrids research: a review of experimental microgrids and test systems," Renewable \& Sustainable Energy Reviews, vol. 15, no. 1, pp. 186-202, 2011. 


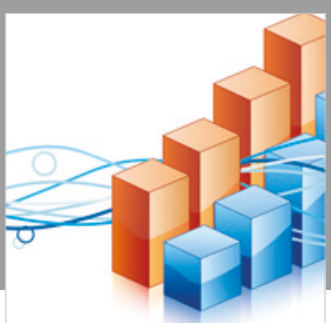

Advances in

Operations Research

\section{-n-m}
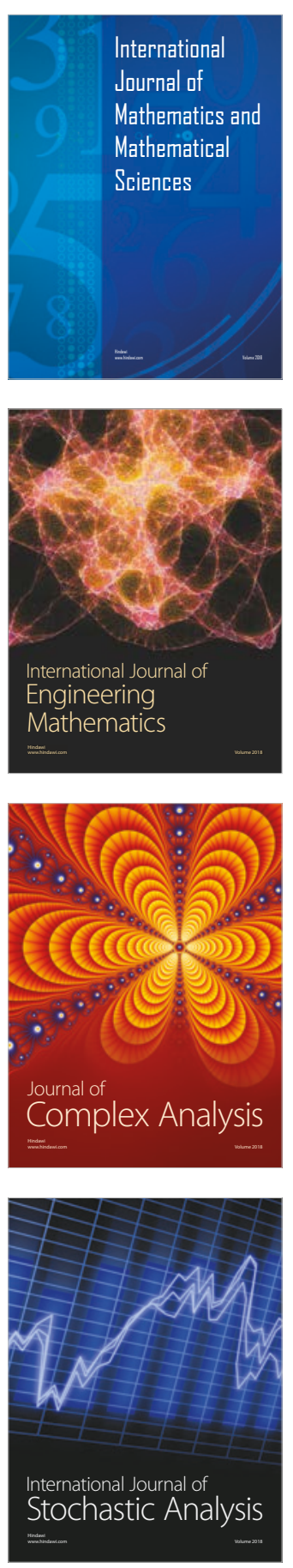
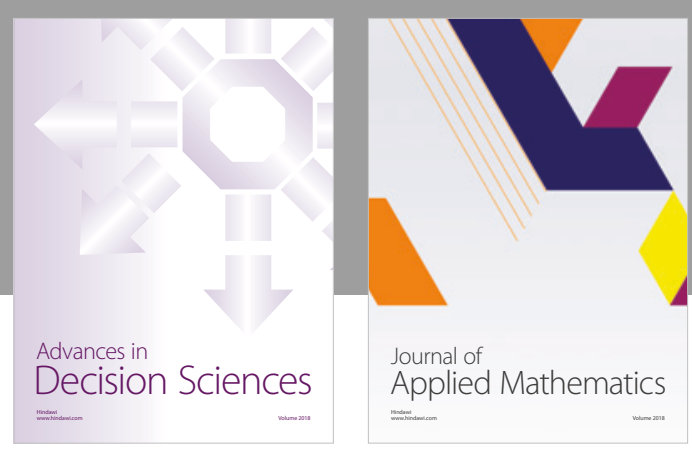

Journal of

Applied Mathematics
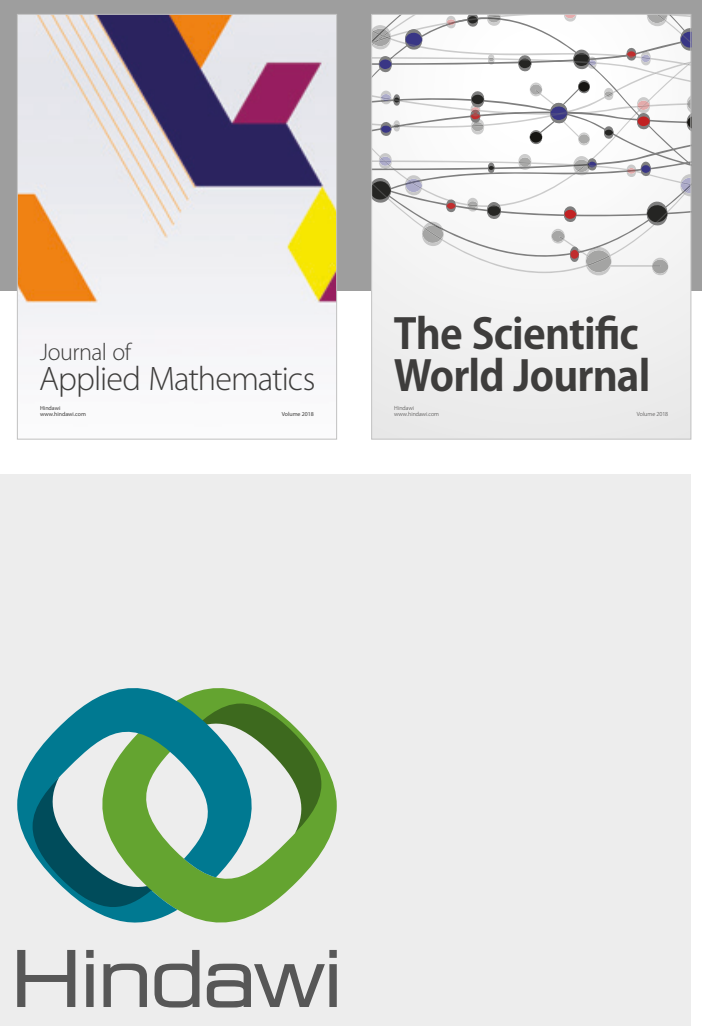

Submit your manuscripts at

www.hindawi.com

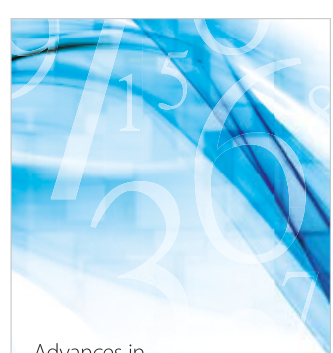

Advances in
Numerical Analysis
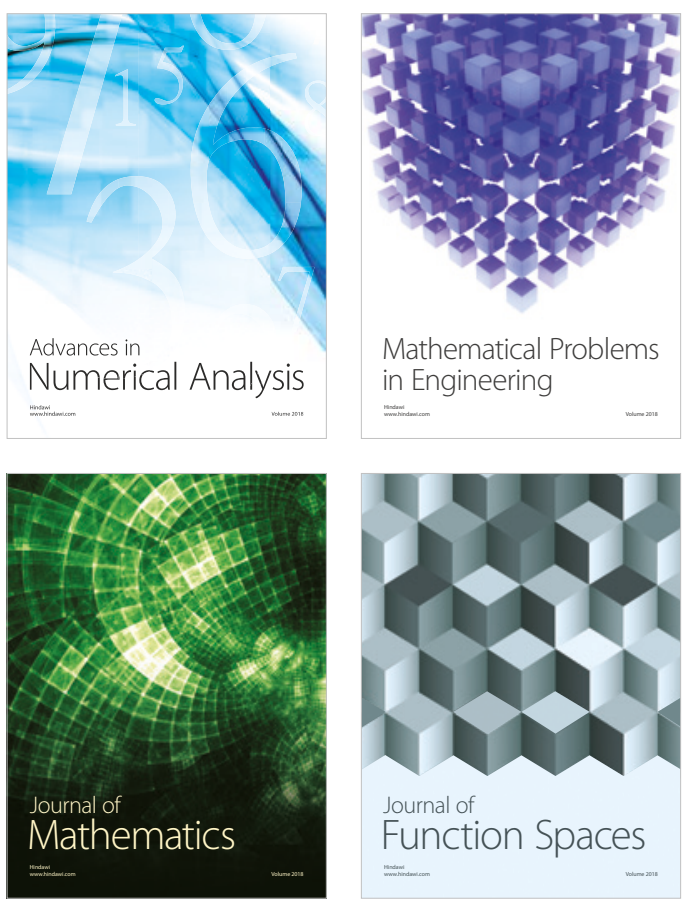

Mathematical Problems in Engineering

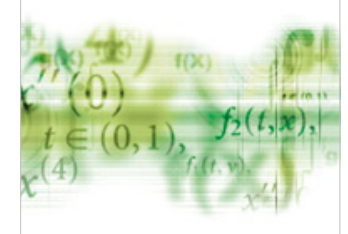

International Journal of

Differential Equations

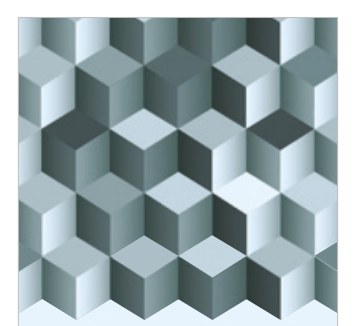

Journal of

Function Spaces

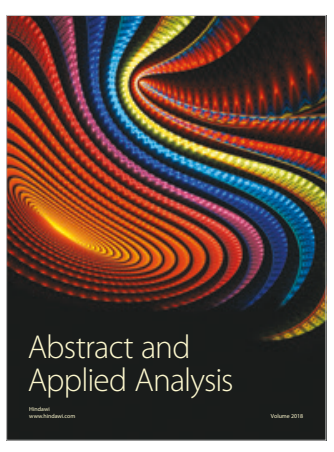

The Scientific

World Journal

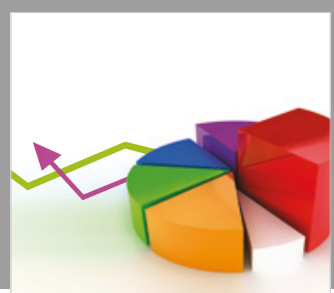

Journal of

Probability and Statistics
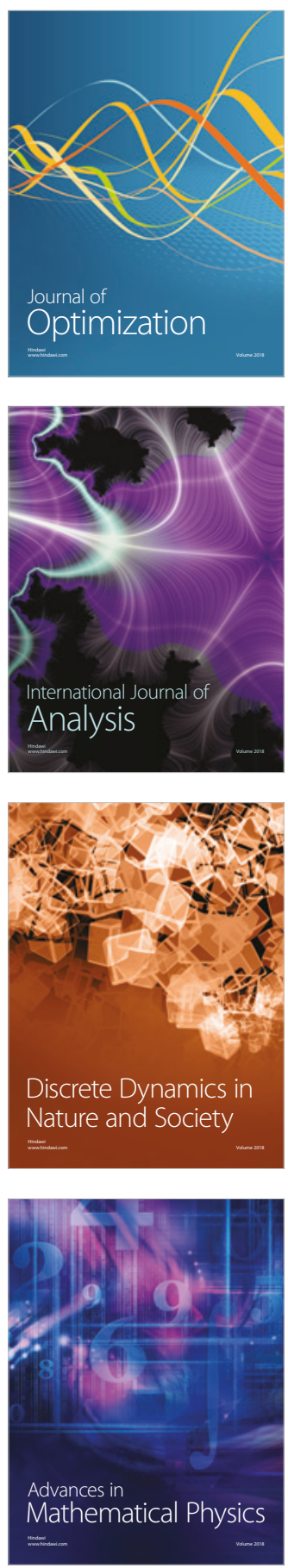